TRANSACTIONS OF THE AMERICAN MATHEMATICAL SOCIETY

Volume 348, Number 4, April 1996

\title{
ANALYSIS OF THE WU METRIC. I: THE CASE OF CONVEX THULLEN DOMAINS
}

\author{
C. K. CHEUNG AND KANG-TAE KIM
}

\begin{abstract}
We present an explicit description of the Wu metric on the convex Thullen domains which turns out to be the first natural example of a purely Hermitian, non-Kählerian invariant metric. Also, we show that the Wu metric on these Thullen domains is in fact real analytic everywhere except along a lower dimensional subvariety, and is $C^{1}$ smooth overall. Finally, we show that the holomorphic curvature of the Wu metric on these Thullen domains is strictly negative where the $\mathrm{Wu}$ metric is real analytic, and is strictly negative everywhere in the sense of current.
\end{abstract}

\section{INTRODUCTION}

1.1. General. One of the main results of this article is that there exists a natural class of invariant non-Kähler Hermitian metrics.

Traditionally, invariant metrics have played an important central role in the study of both differential geometry and several complex variables. A list of the widely studied well-known examples to date consists of the Poincaré-Bergman metric, the Einstein-Kähler metric, the Carathéodory metric and the Kobayashi metric. ([4], [8], [14] to list only a few.) None of these invariant metrics belong to Hermitian geometry properly, as the first two are Kählerian, while the next two are only Finslerian. Although it is agreed in principle that Hermitian geometry is the most natural and general geometry to study in conjunction with the theory of complex analytic functions, the study of this geometry received relatively little attention partly due to the lack of natural examples of non-Kähler Hermitian metrics. We show in this paper that the metric introduced by $\mathrm{H}$. Wu in his article [20] turns out to be the first natural example of non-Kähler Hermitian metrics. Throughout this article, we call this metric the Wu metric. A description of this metric is presented in Section 2 of this article.

The $\mathrm{Wu}$ metric is an upper semicontinuous invariant pseudo-Hermitian metric defined on every complex manifold. It is a complete, continuous Hermitian metric whenever the Kobayashi metric is complete. It is Hermitian in the sense that it is a Hermitian inner product on each tangent space. But, above all, this metric is special since it possesses the distance decreasing property up to a fixed constant factor for any holomorphic mapping between two complex manifolds. This distance decreasing property plays a major role in applying the Kobayashi or Carathéodory

Received by the editors February 6, 1995.

Key words and phrases. Kobayashi metric, invariant Hermitian metric, hyperbolic complex manifold, smoothness, holomorphic curvature.

Research of the second named author is supported in part by grants from Pohang University of Science and Technology and GARC of Seoul National University. 
metric in the study of analytic function theory, and this property is not shared by the aforementioned invariant Kählerian metrics.

Several fundamental questions arise immediately. The first question is whether the $\mathrm{Wu}$ metric is smooth and, if it is, whether it is in fact Kählerian. Then another question is whether the $\mathrm{Wu}$ metric possesses negative holomorphic curvature, since it satisfies an analogue of the distance decreasing property. (See for instance [1], [17], [22], for backgrounds.)

In case the manifold under consideration happens to be homogeneous, in the sense that the group of holomorphic automorphisms acts transitively, the above questions are very easy to answer, as remarked in [20]. However, no further results were known beyond the homogeneous cases. Thus in this article, we explore the above questions on a bounded domain in $\mathbb{C}^{2}$ which is not homogeneous but possesses a noncompact automorphism group. In the light of earlier studies on the bounded domains with noncompact automorphism groups ( [3], [11], [18]), the Thullen domains provide the first nontrivial case for the above questions. The Thullen domains in question are defined by

$$
E_{m}=\left\{\left.\left(z_{1}, z_{2}\right) \in \mathbb{C}^{2}|| z_{1}\right|^{2}+\left|z_{2}\right|^{2 m}<1\right\}
$$

where $m$ is a real number not smaller than $1 / 2$.

It is striking to see that in such a "simple" case the Wu metric already begins to develop a special behavior such as being real analytic outside an explicit singular set. Moreover it shows a well controlled strict negative holomorphic curvature behavior. As a consequence, our results in particular imply a non-Kähler invariant solution of a conjecture of Kobayashi on the Thullen domains which is different from the earlier work of K. Azukawa and M. Suzuki ([2]). We will explain our results more precisely in what follows.

1.2. Main theorems: Smoothness and curvature of the Wu metric. Our first two theorems concern the smoothness of the $\mathrm{Wu}$ metric.

Theorem 1. For $1 / 2 \leq m<1$, the Wu metric $h_{m}$ is real analytic on $\left\{\left(z_{1}, z_{2}\right) \in\right.$ $\left.E_{m} \mid z_{2} \neq 0\right\}$. At the point $(a, 0) \in E_{m}$ for any a with $|a|<1$, the Wu metric is in the Hölder class $C^{1,2 m-1}$, but not in the class $C^{2}$. Moreover, the Wu metric is Hermitian but not Kähler.

For the case $m>1$, we need to decompose $E_{m}$ into the following four disjoint sets:

$$
\begin{aligned}
M^{+} & =\left\{f(0, b) \mid f \in \text { Aut } E_{m}, 2^{-1 / 2 m}<b<1\right\}, \\
M^{0} & =\left\{f\left(0,2^{-1 / 2 m}\right) \mid f \in \text { Aut } E_{m}\right\}, \\
M^{-} & =\left\{f(0, b) \mid f \in \text { Aut } E_{m}, 0<b<2^{-1 / 2 m}\right\}, \\
Z & =\left\{f(0,0) \mid f \in \text { Aut } E_{m}\right\} .
\end{aligned}
$$

Here, Aut $E_{m}$ is the real four dimensional group of biholomorphic automorphisms generated by the complex rotations and the Möbius transformations of the following form:

$$
\left(z_{1}, z_{2}\right) \mapsto\left(\frac{z_{1}+a}{1+\bar{a} z_{1}}, \frac{\left(1-|a|^{2}\right)^{1 / 2 m}}{\left(1+\bar{a} z_{1}\right)^{1 / m}} z_{2}\right)
$$


for $|a|<1$, by taking any branch of the $m$-th root.

Notice that the sets $M^{0}$ and $Z$ are real submanifolds of codimension 1 and 2, respectively, and hence they are "thin" subsets of the domain $E_{m}$. For $m>1$ we have

Theorem 2. For $m>1$, the Wu metric is real analytic everywhere except on a subset of real codimension at least one, while it is at least $C^{1}$ smooth everywhere. More precisely, when $m$ is an integer, the Wu metric $h_{m}$ is real analytic on the open set $E_{m} \backslash M^{0}$, whereas it is only $C^{1}$ smooth at the points in $M^{0}$. If $m$ is not an integer, $h_{m}$ is real analytic on the set $M^{+} \cup M^{-}$, while it is $C^{[2 m]-1}$ smooth at the points in $Z$, and of class $C^{1}$ at the points in $M^{0}$. Also, the Wu metric is Kähler on $M^{+}$but non-Kähler on $M^{-}$.

Explicit expressions of the $\mathrm{Wu}$ metric in these cases are given in Theorems 6 , 7 and 9. This seems to be the first natural explicit example of an invariant Hermitian metric that is neither entirely $C^{\infty}$ smooth, nor Kähler. Also, we would like to remark that the proofs of Theorems 1 and 2 do not depend on brutal calculation. The technical devices that we use to overcome the computational difficulties, square transform and square convexity, could conceivably be useful in other similar situations.

Concerning the holomorphic curvature of the Wu metric of the Thullen domains, we have the following.

Theorem 3. For $1 / 2 \leq m<1$, at every point $\left(z_{1}, z_{2}\right) \in E_{m}$ with $z_{2} \neq 0$, the holomorphic curvature of the Wu metric is bounded from above by $-1 / 2$.

A striking behavior of the holomorphic curvature is observed in case $m>1$, which is

Theorem 4. For every $m>1$, the Wu metric of $E_{m}$ at every point of $M^{+}$is a Kähler metric with constant holomorphic curvature -2. At every point of $M^{-}$, the Wu metric is a non-Kähler Hermitian metric with the holomorphic curvature bounded from above and below by negative constants.

1.3. Negative holomorphic curvature current. A central theme of this article is the study of the following conjecture of Kobayashi.

Conjecture (Kobayashi [14]). On every complex manifold whose Kobayashi metric is complete and proper, there exists a complete Hermitian metric with its holomorphic curvature bounded from above by a negative constant.

It is worth mentioning that $\mathrm{Wu}([20])$ has observed that the following modified conjecture will be equally effective in application.

Conjecture. On every complex manifold whose Kobayashi metric is complete and proper, there exists a $C^{k}$ smooth (for some $k \geq 0$ ) complete Hermitian metric with its holomorphic curvature bounded above by a negative constant in the sense of current.

In this context, we present

Theorem 5. The holomorphic curvature of the Wu metric on the Thullen domain $E_{m}$ is bounded above by a negative constant in the sense of current, for every $m>1 / 2$. 
The derivation of this theorem from Theorems 1 through 4 is rather elementary. We choose to include the proof in an appendix at the end of this article. Notice that Theorem 5 provides a natural answer to Kobayashi's conjecture for the Thullen domains $E_{m}$ with $m \geq 1 / 2$. It suggests that the $\mathrm{Wu}$ metric (or its modification) may be a natural answer to the aforementioned conjecture of Kobayashi.

It is even more striking to see that, for every real number $m>1$, the domain $E_{m}$ admits an explicitly described open neighborhood $\left(M^{+}\right.$as described above $)$ of the strongly pseudoconvex boundary points in which the holomorphic curvature of the $\mathrm{Wu}$ metric is identically -2 . This result is quite impressive compared with the mere asymptotic constancy of the holomorphic curvature of the Bergman and Einstein-Kähler metrics near the strongly pseudoconvex boundary. (See [5], [12], [13].)

The properties and behavior of the $\mathrm{Wu}$ metric in the cases of non-convex Thullen domains and strongly convex smooth domains will be discussed in separate articles.

Acknowledgments. It is our pleasure to acknowledge our indebtedness to $\mathrm{H}$. Wu for his encouragement, interest, and many enlightening discussions with the authors. He also read the first draft of this manuscript and offered us numerous important suggestions. Moreover, the proofs of the properties of the $\mathrm{Wu}$ metric we list in Section 2 of this article are in one of his notes which is not yet published. We are grateful for his generosity in permitting us to use such valuable information.

1.4. Organization of this paper. The organization of this paper from here on is as follows. In Section 2, the construction of the $\mathrm{Wu}$ metric is presented, with a list of several important properties. Section 3 is about the smoothness of the $\mathrm{Wu}$ metric. Finally, Section 4 is about the curvature analysis of the Wu metric of Thullen domains. Along the way, we actually give explicit formulas for the $\mathrm{Wu}$ metric on Thullen domains.

\section{Fundamentals of the Wu metric}

Throughout this section without exception, $\beta_{0}$ denotes the Poincaré-Bergman metric of the unit ball $B^{n}$ in $\mathbb{C}^{n}$ with the constant holomorphic curvature - 4 . Also, a complex manifold $M$ is hyperbolic (in the sense of Kobayashi), if the Kobayashi distance is a proper distance on $M$.

2.1. Construction of the Wu metric. Let $M$ be a complex manifold of complex dimension $n$ and let $x \in M$. Define the set $\mathcal{F}_{x}$ by

$$
\mathcal{F}_{x}:=\left\{f: B^{n} \rightarrow M \mid f \text { holomorphic, } f(0)=x, f_{*}: T_{0} B \rightarrow T_{x} M \text { nonsingular }\right\} .
$$

Then, for each $f \in \mathcal{F}_{x}$ we define a Hermitian inner product $f_{!} \beta_{0}$ on the tangent space $T_{x} M$ of $M$ by

$$
f_{!} \beta_{0}(u, v):=\beta_{0}\left(f_{*}^{-1} u, f_{*}^{-1} v\right), \forall u, v \in T_{x} M .
$$

Now, consider the set

$$
\Psi_{x}=\left\{f_{!} \beta_{0} \mid f \in \mathcal{F}_{x}\right\}
$$

for each $x \in M$, and the set

$$
\Psi=\bigcup_{x \in M} \Psi_{x}
$$


which is of course a biholomorphic invariant of $M$.

Let $\mathcal{Q}_{x}$ be the set of all positive semi-definite Hermitian inner products on $T_{x} M$. In $\mathcal{Q}_{x}$, define the relation $\ll$ by

$$
\alpha \ll \beta \text { if and only if } \alpha(u, u) \leq \beta(u, u), \forall u \in T_{x} M \text {. }
$$

Also define the relation $\prec$ by

$$
\alpha \prec \beta \text { if and only if } \operatorname{det}\left[\alpha\left(u_{i}, u_{j}\right)\right] \leq \operatorname{det}\left[\beta\left(u_{i}, u_{j}\right)\right]
$$

for any choice of a basis $\left\{u_{1}, \ldots, u_{n}\right\}$ for $T_{x} M$. As discussed in [20], the comparison between the determinants is independent of the choice of basis even though the determinant itself is not. Finally, let $\ell\left(\Psi_{x}\right)$ be the set of lower bounds of $\Psi_{x}$ with respect to the partial ordering $\ll$. Then, the following hold:

Lemma 1. $\ell\left(\Psi_{x}\right)$ is a compact subset of $\mathcal{Q}_{x}$ equipped with the usual topology of the space of all sesquilinear forms.

Lemma 2. For every $x$, there exists a unique element $h_{x}$ in $\ell\left(\Psi_{x}\right)$ which satisfies

$$
g_{x} \prec h_{x}, \forall g_{x} \in \ell\left(\Psi_{x}\right) .
$$

Therefore, we arrive at

Definition 1. The Wu metric on a complex manifold $M$ is defined by the assignment

$$
x \mapsto h_{x}: M \rightarrow \mathcal{Q}=\bigcup_{x \in M} \mathcal{Q}_{x}
$$

satisfying $h_{x} \in \mathcal{Q}_{x}$ for each $x \in M$.

2.2. Fundamental properties. The preceding construction shows that the $\mathrm{Wu}$ metric depends only on the complex linear structure of each tangent space. Therefore, it follows immediately that the $\mathrm{Wu}$ metric is an invariant metric, in the sense that every biholomorphic mapping is an isometry with respect to the Wu metrics of the domain and target complex manifolds.

A more intuitive description of the Wu metric is also available, which will play an important role in the later sections of this article. For this, note first the fact that each Hermitian metric is completely determined by its unit ball in each tangent space. Then we arrive at the following geometric description, which is also discussed in $[20]$.

Fix $x \in M$. Endow any Hermitian inner product on $T_{x} M$, and introduce the volume form accordingly. Let $k_{M}$ denote the Kobayashi-Royden infinitesimal metric on $M$. Then, for each positive definite Hermitian inner product $\alpha$ on $T_{x} M$, consider its unit ball

$$
B_{\alpha}=\left\{v \in T_{x} M \mid \alpha(v, v) \leq 1\right\}
$$

and the set

$$
\mathcal{K}_{x}=\left\{v \in T_{x} M \mid k_{M}(x ; v) \leq 1\right\},
$$

commonly called the Kobayashi indicatrix of $M$ at $x$. Denote by $P_{x}$ the set of all positive definite Hermitian inner products on $T_{x} M$. Then, we have 
Proposition $1(\mathrm{Wu})$. The $W u$ metric $h_{x}$ on $T_{x} M$ satisfies

1. $\mathcal{K}_{x} \subseteq B_{h_{x}}$, and

2. volume of $B_{h_{x}} \leq$ volume of $B_{g}$ for every $g \in \mathcal{P}_{x}$ that satisfies $\mathcal{K}_{x} \subseteq B_{g}$, where the volume is measured by any Hermitian inner product on $T_{x} M$.

In other words, the $\mathrm{Wu}$ metric is obtained pointwise by considering the complex ellipsoid of minimum "volume" centered at the origin containing the Kobayashi indicatrix in each tangent plane. The following results are given in [20] without a detailed proof. A proof is given in [21] based upon the arithmetic-geometric mean inequality. We remark that an alternative proof can be obtained by a variation of Fritz John's work [10].

Proposition $2(\mathrm{Wu})$. Let $M, N$ be Kobayashi hyperbolic complex manifolds with the Wu metrics $h_{M}$ and $h_{N}$ respectively. The following hold:

1. The Wu metric is an invariant metric, in the sense that $F^{*} h_{N}=h_{M}$ for any biholomorphic mapping $F: M \rightarrow N$.

2. The Wu metric is a continuous positive definite Hermitian metric.

3. The Wu metric is complete whenever the Kobayashi distance is a complete distance.

4. If $f: M \rightarrow N$ is a holomorphic mapping and $\operatorname{dim} M=n$, then $f^{*} h_{N} \leq$ $\sqrt{n} h_{M}$.

We remark that Lempert's work [16] on convex domains shows that the Kobayashi metric is represented in each complex direction by the push-forward of the Poincaré metric through extremal maps. However, for the Wu metric, such a pointwise representation fails in general. In fact, for any holomorphic mapping $f: B^{n} \rightarrow M$ with $f(0)=x$ and $d f_{0}$ nonsingular, we have

$$
k_{M}(u)^{2}=k_{M}\left(f_{*} w\right)^{2}=f^{*} k_{M}(w)^{2} \leq \beta_{0}(w, w)=\beta_{0}\left(f_{*}^{-1} u, f_{*}^{-1} u\right)=f_{!} \beta_{0}(u, u)
$$

for any $u \in T_{x} M$ and $w \in T_{0} B^{n}$ with $f_{*} w=u$. This shows that the unit ball for the Hermitian inner product $f_{!} \beta_{0}$ in $T_{x} M$ is always a subset of the Kobayashi indicatrix. Consequently, the Wu metric at $x \in M$ cannot coincide with $f_{!} \beta_{0}$ for any holomorphic function $f: B^{n} \rightarrow M$ with $f(0)=x$, unless the Kobayashi-Royden metric at $x$ itself is already Hermitian, which seldom occurs. See, for example, ([7]) and ([16]). Note for instance that the Kobayashi-Royden metric on a convex domain is never Hermitian unless the convex domain itself is biholomorphic to the unit open ball ([16]). This already provides an infinite dimensional family of holomorphically inequivalent convex domains ([7]) on which the Kobayashi-Royden metric is not Hermitian, consequently on which the $\mathrm{Wu}$ metric cannot admit pointwise representation as explained above.

\section{Explicit Description and Smoothness on Thullen Domains}

3.1. Preliminaries for the Kobayashi-Royden metric. We first introduce some basic facts about the Kobayashi-Royden metric in the Thullen domain $E_{m}=$ $\left\{\left.\left(z_{1}, z_{2}\right) \in \mathbb{C}^{2}|| z_{1}\right|^{2}+\left|z_{2}\right|^{2 m}<1\right\}$ where $m$ is a real number $>1 / 2$. Since the holomorphic tangent bundle of the Thullen domain $E_{m}$ is trivial, we identify from now on the tangent bundle $T E_{m}$ of $E_{m}$ with $E_{m} \times \mathbb{C}^{2}$ and use the standard Euclidean coordinates $(z ; v)=\left(z_{1}, z_{2} ; v_{1}, v_{2}\right)$ throughout this article. 
According to [6] and [9], we have the following explicit expression for the square of the Kobayashi-Royden metric at $(0, b)$ in the direction $v=\left(v_{1}, v_{2}\right)$ :

$$
\begin{aligned}
k^{2}((0, b) ; v) & =\frac{\left|v_{1}\right|^{2}}{1-|b|^{2 m}}+\frac{m^{2}|b|^{2 m-2}\left|v_{2}\right|^{2}}{\left(1-|b|^{2 m}\right)^{2}}, \text { for } u=m\left|v_{2} / v_{1}\right| \leq|b|, \\
k^{2}((0, b) ; v) & =\frac{\left|v_{2}\right|^{2} t^{2} m^{2}\left(1-a^{2}\right)^{2}}{|b|^{2}\left(t^{2}-1\right)^{2}\left(m\left(1-a^{2}\right)+a^{2}\right)^{2}} \\
& =\frac{a^{2} t^{2}\left|v_{1}\right|^{2}}{\left(1-t^{2 m}|b|^{2 m}\right)^{2}}, \text { for } u=m\left|v_{2} / v_{1}\right|>|b|,
\end{aligned}
$$

where $a$ and $t$ are defined implicitly as follows: the term $a$ by

$$
a=\frac{2 m|b|}{u+\left(u^{2}+4 m(m-1)|b|^{2}\right)^{1 / 2}}
$$

and the term $t$ by the unique positive solution of the equation

$$
t^{2} a^{2}+t^{2 m}\left(1-a^{2}\right)|b|^{2 m}=1
$$

Here, notice that one obtains

$$
0<a<1 \text {, and } 1<t<1 /|b|
$$

It is relatively straightforward to confirm that the above formulae for the square of the Kobayashi metric have natural continuation to the cases such as $b=0$ and $v_{1}=0$. See [6] for details. Furthermore, away from the zero section of the tangent bundle of the Thullen domain $E_{m}$, both expressions (2) and (3) are smooth in $b$, separately, as $b$ ranges over the open unit disk in $\mathbb{C}$. In the tangent space at $(0, b)$, it is shown in [6] that the Kobayashi metric is $C^{1}$ smooth at the point $v=\left(v_{1}, v_{2}\right) \neq(0,0)$ with $m\left|v_{2}\right|=|b|\left|v_{1}\right|$. Since the action of the automorphism group of biholomorphisms of the Thullen domain $E_{m}$ is real analytic and every point of $E_{m}$ is in the orbit of the point $(0, b)$ for some $b$ with $0 \leq b<1$, these establish the $C^{1}$ smoothness of the Kobayashi-Royden metric off the zero section of the tangent bundle. However, no further investigation on further differentiability was attempted in [6], perhaps due to the complexity of the computation. We will show in later sections by a simpler method, which is a by-product of our methods, that at the point $v=\left(v_{1}, v_{2}\right) \neq(0,0)$ in the tangent space $T_{(0, b)} E_{m}=\mathbb{C}^{2}$ with $m\left|v_{2}\right|=|b|\left|v_{1}\right|$ for every $(0, b) \in E_{m}$ the Kobayashi-Royden metric is in fact $C^{2}$ but not $C^{3}$ smooth. For the proof, see Sections 3.2.2 and 4.2.2.

It follows by the explicit expression of the Kobayashi-Royden metric $k^{2}(v)$ of the Thullen domain above ([6]) that at each reference point $(0, b) \in E_{m}$ the Kobayashi metric is a function of $\left|v_{1}\right|$ and $\left|v_{2}\right|$. Therefore, the $\mathrm{Wu}$ metric constructed from the Kobayashi metric takes a very special form at the reference point $(0, b)$ in this coordinate system. To observe this, recall first that the Wu metric in our coordinate system above is given by the ellipsoid of the minimum Euclidean volume containing the Kobayashi indicatrix in the tangent space at each reference point in the domain $E_{m}$. We will call such an ellipsoid the best fitting (complex) ellipsoid. Fix $z \in E_{m}$ and let the unit sphere of the $\mathrm{Wu}$ metric in $T_{z} E_{m}$ be represented by

$$
r_{1}\left|v_{1}\right|^{2}+r_{2}\left|v_{2}\right|^{2}+2 \operatorname{Re} r_{3} v_{1} \bar{v}_{2}=1,
$$


where $r_{1}, r_{2}>0$ and $r_{3} \in \mathbb{C}$. Notice that this ellipsoid is not invariant under the action of the group

$$
T^{2}=\left\{\left(v_{1}, v_{2}\right) \mapsto\left(e^{i \theta} v_{1}, e^{i \psi} v_{2}\right) \mid \theta, \psi \in \mathbb{R}\right\}
$$

unless $r_{3}=0$. Therefore, if $r_{3} \neq 0$, the Kobayashi indicatrix in $T_{(0, b)} E_{m}$ would then admit more than one best fitting ellipsoid, because the Kobayashi indicatrix $\mathcal{K}_{(0, b)}$ is invariant under the action by $T^{2}$ and this action is Euclidean volume preserving. This contradicts Lemma 2 on the uniqueness of the Wu metric, which is in this context the uniqueness of the best fitting ellipsoid. (See also [10].) Therefore, combining this with the basic results on the $\mathrm{Wu}$ metric of the preceding section, we arrive at

Proposition 3. In terms of Euclidean coordinates on the tangent bundle $E_{m} \times$ $\mathbb{C}^{2}=T E_{m}$ of the Thullen domain $E_{m}$, for every $(0, b) \in E_{m}$ the unit sphere of the Wu metric in $T_{(0, b)} E_{m}$ is of the form

$$
r_{1}\left|v_{1}\right|^{2}+r_{2}\left|v_{2}\right|^{2}=1,
$$

where $r_{1}$ and $r_{2}$ are positive real-valued continuous functions of $b$.

At this point, it is convenient to introduce the following terminology and notation for later purposes.

Definition 2. Let $(0, b) \in E_{m}$. Restrict $v_{1}, v_{2}$ to the positive real axis. Then, let $C_{\text {low }}$ denote the curve $k^{2}=1$ in the first quadrant of $\mathbb{R}^{2}$, with $k^{2}$ given by (2) above, which we call the lower $K$-curve. In turn, we define the upper $K$-curve $C_{u p}$ to be the curve $k^{2}=1$ where $k^{2}$ in this case is given by (3).

Notice in particular that the expressions of the upper curve $C_{u p}$ and the lower curve $C_{\text {low }}$ are $C^{\infty}$ smooth function in the variable $b$, for all $b$ with $0<|b|<1$. In case $m$ is a positive integer, they extend smoothly across $b=0$. We shall show later how this leads to the $C^{\infty}$ smoothness of the Wu metric of the Thullen domains.

3.2. Square convexity of the K-curves. Throughout this section, we assume

$$
0<b<1 \text {. }
$$

To investigate the differentiability of the Wu metric which is now the solutions of the extremal problem with respect to finding a parameterized family of best fitting regular ellipsoids, we introduce the concept of square convexity as follows:

Definition 3. Let $f:\left(t_{0}, t_{1}\right) \rightarrow \mathbb{R}$ be a strictly decreasing positive real-valued function defined on the interval $\left(t_{0}, t_{1}\right)$ with $t_{0} \geq 0$. The function $f$ is said to be square-concave if

$$
\frac{d^{2}\left(f(\sqrt{s})^{2}\right)}{d s^{2}} \leq 0 \text { for all } s \in\left(t_{0}^{2}, t_{1}^{2}\right) .
$$

If the second derivative is strictly negative, we call $f$ strictly square-concave. Likewise, we may define the square-convexity and strict square-convexity if the second derivative above is positive.

Now we state the main result of this section. Let us denote by $v_{2}=f_{u p}\left(v_{1}\right)$ the function representing the graph of the upper K-curve $C_{u p}$ in the first quadrant of $\mathbb{R}^{2}$, and by $v_{2}=f_{\text {low }}\left(v_{1}\right)$ the function representing the lower K-curve, respectively. (See Definition 2 for the terminology.) Then we have 
Proposition 4. $f_{\text {low }}$ is always both square-convex and square-concave. $f_{u p}$ is strictly square-concave if $m>1$, and strictly square-convex if $1 / 2 \leq m<1$.

Proof. The first statement is obvious, since $C_{l o w}$ is part of an ellipse centered at the origin. Notice that under the coordinate change $\left(v_{1}, v_{2}\right) \mapsto\left(v_{1}^{2}, v_{2}^{2}\right)$ all regular ellipses centered at the origin become straight lines in the first quadrant. This implies that

$$
\frac{d^{2}\left(f_{l o w}(\sqrt{s})^{2}\right)}{d s^{2}}=0
$$

To prove the second and third statements, we will derive a parametric equation for $f_{u p}$ in terms of $t$ from (3), (4) and (5). Here we use the notation in 3.1. Rewrite (5) as

$$
a^{2}=\frac{1-t^{2 m}|b|^{2 m}}{t^{2}-t^{2 m}|b|^{2 m}} .
$$

Then,

$$
1-a^{2}=\frac{t^{2}-1}{t^{2}-t^{2 m}|b|^{2 m}} .
$$

To describe $f_{u p}$ from the curve $C_{u p}: k^{2}=1$ with $k^{2}$ as in (3), we have

$$
\frac{a t\left|v_{1}\right|}{1-t^{2 m}|b|^{2 m}}=1
$$

and

$$
\frac{\left|v_{2}\right| t m\left(1-a^{2}\right)}{|b|\left(t^{2}-1\right)\left(m\left(1-a^{2}\right)+a^{2}\right)}=1
$$

After substituting the expressions of $a^{2}$ and $1-a^{2}$ into these equations, we arrive at the parametric equation for $v_{2}=f_{u p}\left(v_{1}\right)$ representing the upper K-curve $C_{u p}$ at $(0, b) \in E_{m}$ in terms of $t$ with $b>0$ as follows:

$$
\left\{\begin{array}{l}
v_{1}=\frac{1}{t} \sqrt{1-t^{2 m} b^{2 m}} \sqrt{t^{2}-t^{2 m} b^{2 m}}, \\
v_{2}=\frac{b}{m t}\left(m t^{2}-m+1-t^{2 m} b^{2 m}\right), \quad 1<t<1 / b .
\end{array}\right.
$$

To show the square-convexity or concavity of the function $v_{2}=f_{u p}\left(v_{1}\right)$, it suffices to check the sign of the expression

$$
A=\frac{x^{\prime}(t) y^{\prime \prime}(t)-x^{\prime \prime}(t) y^{\prime}(t)}{\left(x^{\prime}(t)\right)^{3}}, 1<t<1 / b,
$$

where

$$
\left\{\begin{array}{l}
x(t)=\left(v_{1}(t)\right)^{2}=\left(1-t^{2 m} b^{2 m}\right)\left(1-t^{2 m-2} b^{2 m}\right), \\
y(t)=\left(v_{2}(t)\right)^{2}=\frac{b^{2}}{m^{2} t^{2}}\left(m t^{2}-m+1-t^{2 m} b^{2 m}\right)^{2}
\end{array}\right.
$$

for all $m \geq 1 / 2$.

First we show $x^{\prime}(t)$ is never zero for all $t \in(1,1 / b)$. A direct computation yields

$$
\begin{aligned}
x^{\prime}(t) & =-2 t^{2 m-3} b^{2 m}\left(m t^{2}+m-1-(2 m-1) t^{2 m} b^{2 m}\right) \\
& <-2 m t^{2 m-3} b^{2 m}\left(t^{2}-1\right) \\
& <0 .
\end{aligned}
$$


Now, we investigate the sign of $A$ from calculating $N$, the denominator of $A$ for $1<t<1 / b$, explicitly. First,

$$
x^{\prime}(t)=2 t^{2 m-3} b^{2 m} \cdot E,
$$

where

$$
E=(2 m-1) t^{2 m} b^{2 m}-m+1-m t^{2} .
$$

Before computing the derivatives of $y(t)$, let

$$
F=m t^{2}-m+1-t^{2 m} b^{2 m} .
$$

Then,

$$
y^{\prime}(t)=-2 b^{2} m^{-2} t^{-3} \cdot F \cdot E .
$$

For the second derivative, we get

$$
y^{\prime \prime}(t)=-2 b^{2} m^{-2} t^{-4}\left[\left(t F^{\prime}-3 F\right) E+t F E^{\prime}\right] .
$$

Combining all these, we obtain

$$
\begin{aligned}
N & =x^{\prime}(t) y^{\prime \prime}(t)-x^{\prime \prime}(t) y^{\prime}(t) \\
& =8 b^{2 m+2} t^{2 m-7} m^{-1} \cdot E^{2} \cdot\left(t^{2}-1\right)(m-1) .
\end{aligned}
$$

In fact, there are simple geometric reasons why the preceding factorization should occur. When $m=1$, the Thullen domain $E_{1}$ is the unit ball, whose Kobayashi metric is indeed Hermitian. In such a case $N$ vanishes, since every regular ellipsoid centered at the origin is square-convex and concave at the same time. This suggests that $m-1$ is a factor of $N$. The reason why $t^{2}-1$ should be a factor is that $t^{2}=1$ corresponds to the case when $b=1$. As $b \rightarrow 1-$, it turns out that the whole Kobayashi indicatrix "converges" to the extension of the ellipsoid defined by equating the expression (2) with 1. Again, the lower K-curve is square-convex and concave at the same time, and so one may expect that $t^{2}-1$ should be a factor.

Now, combining (9), (10), (11) and (12), we deduce that the function $f_{u p}$ is strictly square-concave if $m>1$, and strictly square-convex if $1 / 2 \leq m<1$. Therefore, the proof of the proposition is now complete.

3.3. Wu metric on $E_{m}$ for $1 / 2 \leq m<1$. We will first compute the $\mathrm{Wu}$ metric tensor at the point $(0, b) \in E_{m}$, where $0<b<1$. Recall that under the coordinate transform $(x, y) \mapsto\left(x^{2}, y^{2}\right)$ all the regular ellipses in $\mathbb{R}^{2}$ centered at the origin become a straight line segment in the first quadrant. Since the upper $\mathrm{K}$-curve $C_{u p}$ and the lower $\mathrm{K}$-curve $C_{\text {low }}$ form together a curve which is at least $C^{1}$ smooth ([6]), obviously this curve will stay $C^{1}$ smooth even after the coordinate transform $(x, y) \mapsto\left(x^{2}, y^{2}\right)$ of the first quadrant. Thus, Proposition 4 implies that the curves transformed from $C_{u p}$ and $C_{\text {low }}$, under the aforementioned coordinate transform, together form a $C^{1}$ smooth convex curve. Therefore, the straight line segment in the first quadrant which encloses the smallest area with the two axes containing this $C^{1}$ smooth convex curve in its interior has to be the line segment joining the $v_{2}$-intercept of the transformed upper $K$-curve $C_{u p}$ and the $v_{1}$-intercept of the transformed lower K-curve $C_{\text {low }}$. Consequently, by Proposition 3 it is easy 
to see that the best fitting complex ellipsoid, which is the $\mathrm{Wu}$ metric sphere at $(0, b) \in E_{m}$, is given by

$$
\begin{aligned}
h_{1 \overline{1}}(0, b) & =\frac{1}{1-b^{2 m}}, \\
h_{1 \overline{2}}(0, b) & =\bar{h}_{2 \overline{1}}(0, b)=0, \\
h_{2 \overline{2}}(0, b) & =\frac{1}{\left(1-b^{2}\right)^{2}} .
\end{aligned}
$$

Now, using the invariance of the $\mathrm{Wu}$ metric under the action of the automorphisms of $E_{m}$ such as

$$
\left(z_{1}, z_{2}\right) \rightarrow\left(\frac{z_{1}-p_{1}}{1-\bar{p}_{1} z_{1}}, \frac{\left|p_{2}\right|}{p_{2}} \cdot \frac{\left(1-\left|p_{1}\right|^{2}\right)^{1 / 2 m} z_{2}}{\left(1-\bar{p}_{1} z_{1}\right)^{1 / m}}\right)
$$

which maps $\left(p_{1}, p_{2}\right)$ with $p_{2} \neq 0$ to $\left(0,\left|p_{2}\right|\left(1-\left|p_{1}\right|^{2}\right)^{-1 / 2 m}\right)$, and using the continuity of the Wu metric (Proposition 2), we arrive at the following explicit formula for the Wu metric tensor.

Theorem 6. The Wu metric of the Thullen domain $E_{m}$ with $1 / 2 \leq m<1$ in the coordinates $\left(p_{1}, p_{2} ; v_{1}, v_{2}\right)$ for $E_{m} \times \mathbb{C}^{2}=T E_{m}$ is given by

$(15)$

$$
\begin{aligned}
h_{m}= & \left(\frac{1}{\left(1-\left|p_{1}\right|^{2}\right)\left(1-\left|p_{1}\right|^{2}-\left|p_{2}\right|^{2 m}\right)}+\frac{\left(1-\left|p_{1}\right|^{2}\right)^{-2+1 / m}\left|p_{1}\right|^{2}\left|p_{2}\right|^{2}}{m^{2}\left(\left(1-\left|p_{1}\right|^{2}\right)^{1 / m}-\left|p_{2}\right|^{2}\right)^{2}}\right) d p_{1} \otimes d \bar{p}_{1} \\
& +\frac{\left(1-\left|p_{1}\right|^{2}\right)^{-1+1 / m} \bar{p}_{1} p_{2}}{m\left(\left(1-\left|p_{1}\right|^{2}\right)^{1 / m}-\left|p_{2}\right|^{2}\right)^{2}} d p_{1} \otimes d \bar{p}_{2} \\
& +\frac{\left(1-\left|p_{1}\right|^{2}\right)^{-1+1 / m} p_{1} \bar{p}_{2}}{m\left(\left(1-\left|p_{1}\right|^{2}\right)^{1 / m}-\left|p_{2}\right|^{2}\right)^{2}} d p_{2} \otimes d \bar{p}_{1} \\
& +\frac{\left(1-\left|p_{1}\right|^{2}\right)^{1 / m}}{\left(\left(1-\left|p_{1}\right|^{2}\right)^{1 / m}-\left|p_{2}\right|^{2}\right)^{2}} d p_{2} \otimes d \bar{p}_{2}
\end{aligned}
$$

From the explicit description of the $\mathrm{Wu}$ metric in the above, it is easy to see that the $\mathrm{Wu}$ metric is real analytic except when $p_{2}=0$. In particular, the proof of Theorem 1 follows immediately. It is also simple to observe that the Wu metric is not $C^{2}$ smooth at the points $\left(p_{1}, 0\right) \in E_{m}$ for any $p_{1}$ with $\left|p_{1}\right|<1$, although it is of Hölder class $C^{1,2 m-1}$. Moreover, a direct computation shows that the $\mathrm{Wu}$ metric given above is not Kähler. This fact is also observed in the analysis of the curvature tensor in Section 4 of this article.

3.4. Wu metric of $E_{m}$ for $m>1$. For the description of the Wu metric tensor in this case, we need to decompose the Thullen domain $E_{m}$ into the four sets $M^{+}, M^{-}, M^{0}$ and $Z$ as described in Section 1.2. Notice that $E_{m}$ is the disjoint union of these four sets, and that $M^{0}$ and $Z$ are low dimensional submanifolds of codimension 1 and 2 respectively, while $M^{+}$and $M^{-}$are open subsets. Thus, we will first give an explicit description of the Wu metric and its smoothness on $\mathrm{M}^{+}$ and $M^{-}$. Then, we will discuss how the $\mathrm{Wu}$ metric extends across the thin sets $M^{0}$ and $Z$. 
3.4.1. Wu metric at $\left(p_{1}, p_{2}\right) \in M^{+}$. Keeping in mind the definition of $M^{+}$in the above, we will first concentrate on the Wu metric at $(0, b) \in E_{m}$ with $2^{-1 / 2 m}<$ $b<1$ and its smooth dependence on $b$. In fact, we are going to show that the unit ball of the Wu metric in $T_{(0, b)} E_{m}$ in this case is given by the extension of the lower K-curve defined by

$$
\frac{\left|v_{1}\right|^{2}}{1-b^{2 m}}+\frac{m^{2} b^{2 m-2}\left|v_{2}\right|^{2}}{\left(1-b^{2 m}\right)^{2}}=1
$$

Recall that the Kobayashi-Royden metric in each tangent space of $E_{m}$ is given in a two piece formula, as in (2) and (3) of Section 3.1. In order to obtain an explicit formula for the $\mathrm{Wu}$ metric, we first compute the "joining point" of the upper and lower K-curves $C_{u p}$ and $C_{\text {low }}$ (see Definition 2 in Section 3.1), which is the point $v=\left(v_{1}, v_{2}\right)$ satisfying

$$
k(v)=1 \quad \text { and } \quad m\left|v_{2}\right|=b\left|v_{1}\right| .
$$

A direct calculation shows that the joining point in the first quadrant of $\mathbb{R}^{2}$ is given by $\left(1-b^{2 m}, b\left(1-b^{2 m}\right) / m\right)$. Now consider the following affine rescaling in the tangent space $\mathbb{C}^{2}=T_{(0, b)} E_{m}$, defined by

$$
\left(v_{1}, v_{2}\right) \mapsto\left(\frac{v_{1}}{\sqrt{1-b^{2 m}}}, \frac{m b^{m-1} v_{2}}{1-b^{2 m}}\right)
$$

Under this rescaling, the lower K-curve transforms to part of the unit circle centered at the origin. Notice that the minimal ellipsoid stays minimal through affine rescaling. When we pass to the coordinate transform $\left(v_{1}, v_{2}\right) \mapsto\left(v_{1}^{2}, v_{2}^{2}\right)$ of the first quadrant of $\mathbb{R}^{2}$, the whole $\mathrm{K}$-curve (upper and lower curves joined at the joining point) becomes a graph of a concave function defined on part of the positive $v_{1}$-axis bounding a convex region together with the coordinate axes. More importantly, the lower K-curve now becomes a straight line segment of slope -1. Furthermore, the joining point between upper and lower curves now transforms to the point $\left(\left(1-b^{2 m}\right)^{1 / 4}, \sqrt{b^{m}}\right)$. Since $1 / 2<b^{2 m}<1$ in our case, it is obvious that the best fitting ellipsoid corresponding to the unit sphere of the Wu metric is now the straight line segment of slope -1 which is the extension of the low K-curve transformed by the aforementioned coordinate change $\left(v_{1}, v_{2}\right) \mapsto\left(v_{1}^{2}, v_{2}^{2}\right)$ in the first quadrant of $\mathbb{R}^{2}$. Thus we obtain the following key lemma:

Lemma 3. The minimum ellipsoid which gives the $W u$ metric is given by the extension of the lower $K$-curve if $b \geq 2^{-1 / 2 m}$.

Consequently, at $(0, b) \in M^{+}$with $b>2^{-1 / 2 m}$, the Wu metric is given by

$$
\begin{aligned}
& g_{1 \overline{1}}(0, b)=\frac{1}{1-b^{2 m}}, \\
& g_{1 \overline{2}}(0, b)=0 \\
& g_{2 \overline{1}}(0, b)=0, \\
& g_{2 \overline{2}}(0, b)=\frac{m^{2} b^{2 m-2}}{\left(1-b^{2 m}\right)^{2}} .
\end{aligned}
$$

Due to the real analytic action of Aut $E_{m}$ on $M^{+}$, this already proves that the $\mathrm{Wu}$ metric is real analytic at every point $p=\left(p_{1}, p_{2}\right) \in M^{+}$. However, for the 
sake of completeness and for later purposes, we present in the following an explicit expression of the $\mathrm{Wu}$ metric tensor using the automorphism

$$
f:\left(z_{1}, z_{2}\right) \mapsto\left(\frac{z_{1}-p_{1}}{1-\bar{p}_{1} z_{1}}, \frac{\left|p_{2}\right|}{p_{2}} \cdot \frac{\left(1-\left|p_{1}\right|^{2}\right)^{1 / 2 m} z_{2}}{\left(1-\bar{p}_{1} z_{1}\right)^{1 / m}}\right),
$$

which maps $\left(p_{1}, p_{2}\right)$ with $p_{2} \neq 0$ to $\left(0,\left|p_{2}\right|\left(1-\left|p_{1}\right|^{2}\right)^{-1 / 2 m}\right)$.

Theorem 7. The Wu metric tensor of the Thullen domain $E_{m}$ for $m>1$ at $\left(p_{1}, p_{2}\right) \in M^{+}$is given by

$$
\sum_{\alpha, \beta=1}^{2} g_{\alpha \bar{\beta}}\left(p_{1}, p_{2}\right) d p_{1} \otimes \overline{d p_{2}}
$$

where

$$
\begin{aligned}
g_{1 \overline{1}}\left(p_{1}, p_{2}\right) & =\frac{1-\left|p_{2}\right|^{2 m}}{\left(1-\left|p_{1}\right|^{2}-\left|p_{2}\right|^{2 m}\right)^{2}}, \\
g_{1 \overline{2}}\left(p_{1}, p_{2}\right) & =\frac{m\left|p_{2}\right|^{2 m-2} \bar{p}_{1} p_{2}}{\left(1-\left|p_{1}\right|^{2}-\left|p_{2}\right|^{2 m}\right)^{2}}, \\
g_{2 \overline{1}}\left(p_{1}, p_{2}\right) & =\overline{g_{1 \overline{2}}}\left(p_{1}, p_{2}\right), \\
g_{2 \overline{2}}\left(p_{1}, p_{2}\right) & =\frac{m^{2}\left|p_{2}\right|^{2 m-2}\left(1-\left|p_{1}\right|^{2}\right)}{\left(1-\left|p_{1}\right|^{2}-\left|p_{2}\right|^{2 m}\right)^{2}} .
\end{aligned}
$$

Later in Section 4.3.1, we will show that the holomorphic curvature of the above tensor is in fact the constant -2 .

3.4.2. Wu metric at $\left(p_{1}, p_{2}\right) \in M^{-}$. We are now interested in the smooth dependence of the $\mathrm{Wu}$ metric tensor at $(0, b)$ on the real variable $b$ when $0<b<2^{-1 / 2 m}$. In this case, it is also possible to obtain an explicit expression for the Wu metric, which we give at the end of this section. However, we will take a more general approach based upon the study of extremal problems by Fritz John in [10]. We take such an approach here because this method can also be applied to the thin sets $M^{0}$ and $Z$.

Since the volume of the unit ball of the Wu metric in Proposition 3 is $\pi /\left(2 r_{1} r_{2}\right)$, we may reformulate the regularity problem of the $\mathrm{Wu}$ metric of a Thullen domain $E_{m}$ as follows:

(*) Investigate the smooth dependence on the real variable $b$ of the minimum point of the function

$$
F\left(r_{1}, r_{2}\right)=-r_{1} r_{2} \text { for } r_{1}, r_{2}>0
$$

under the constraint

$$
G_{b}\left(r_{1}, r_{2} ; v_{1}, v_{2}\right)=\left\{k_{(0, b)}\left(v_{1}, v_{2}\right)\right\}^{2}-r_{1}\left|v_{1}\right|^{2}-r_{2}\left|v_{2}\right|^{2} \geq 0, \forall v \in S_{b},
$$

where $k_{(0, b)}\left(v_{1}, v_{2}\right)$ denotes the Kobayashi-Royden metric of the Thullen domain $E_{m}$ evaluated at $\left(0, b ; v_{1}, v_{2}\right) \in T E_{m}=E_{m} \times \mathbb{C}^{2}$, and where $S_{b}$ is the boundary of a certain compact neighborhood of the origin in $\mathbb{C}^{2}$. 
In fact, the constraint in the above should be given for all $v \in \mathbb{C}^{2}$, but the homogeneity of the function $G_{b}$ in $v$ enables us to replace $\mathbb{C}^{2}$ in the constraint equation by a compact set such as $S_{b}$. Incidentally, note also that due to the same reasoning, we have tremendous freedom in choosing an appropriate $S_{b}$ because the minimum point of the problem $(*)$ above stays invariant even if we change $S_{b}$ for every $b$.

In the following, we will follow Fritz John's method ([10]), with a subtle difference that our choice for $S_{b}$ will also vary with $b$. This becomes important in the later analysis of the regularity of $r(b)$.

Suppose that $r=\left(r_{1}, r_{2}\right)$ is a solution of the extremal problem $(*)$ above. Note that by (2) and (3) of Section 3.1, the functions $k_{(0, b)}\left(v_{1}, v_{2}\right)$ and $G_{b}\left(r_{1}, r_{2} ; v_{1}, v_{2}\right)$ depend only on $\left|v_{1}\right|$ and $\left|v_{2}\right|$. Hence, instead of the problem $(*)$ above, we may consider the following simplified but equivalent problem:

(**) Investigate the regularity of the $\mathbb{R}^{2}$-valued function $r=r(b)$ which yields the minimum of the function

$$
F\left(r_{1}, r_{2}\right)=-r_{1} r_{2} \text { for } r_{1}, r_{2}>0
$$

under the constraint

$$
G_{b}(r, v)=K(b, v)-\left(r_{1} v_{1}^{2}+r_{2} v_{2}^{2}\right) \geq 0
$$

for all $v=\left(v_{1}, v_{2}\right) \in S=S_{b} \subset \mathbb{R}^{2}$, where $S=S_{b}$ is a simple closed curve (depending upon the parameter $b$ in general) in $\mathbb{R}^{2}$ containing the origin inside, and where

$$
K(b, v)=K(b,|v|)=k_{(0, b)}(v)^{2}, \text { for } v=\left(v_{1}, v_{2}\right) \in \mathbb{C}^{2} .
$$

The most crucial step in Fritz John's analysis of the necessary conditions on the above extremal problem is the following:

Lemma 4. Let $\hat{r}$ be a solution of $(* *)$ above, and let $S^{\prime}=\left\{v \in S_{b} \mid G_{b}(\hat{r}, v)=0\right\}$. Then, the intersection of the two sets

$$
\left\{\left(u_{1}, u_{2}\right) \in \mathbb{R}^{2} \mid \frac{\partial F}{\partial r_{1}}(\hat{r}) u_{1}+\frac{\partial F}{\partial r_{2}}(\hat{r}) u_{2}<0\right\}
$$

and

$$
\left\{\left(u_{1}, u_{2}\right) \in \mathbb{R}^{2} \mid \frac{\partial G_{b}}{\partial r_{1}}(\hat{r}, v) u_{1}+\frac{\partial G_{b}}{\partial r_{2}}(\hat{r}, v) u_{2}>0, \forall v \in S^{\prime}\right\}
$$

is empty.

While referring to [10] for a precise proof of this lemma, we give a rough but more intuitive recapitulation of the actual proof. In the statement of the lemma above, the vectors in the first set represent the directions along which the directional derivative is negative. Thus they represent the directions along which the value of the function $F$ is decreasing. On the other hand, those in the second set represent the directions along which the value of $G$ is increasing. Hence, any common element of the two sets above would represent a direction which will further decrease the 
value of $F$ while satisfying the condition $G>0$. Since this obviously cannot happen to the solution of the minimum problem $(* *)$, the conclusion of the lemma follows.

Now, if one interprets the conclusion of Lemma 4 above again, it simply says that the (possibly infinitely many) gradient vectors $-\nabla_{r} F(\hat{r})$ and $\nabla_{r} G_{b}(\hat{r}, v)$ for all $v \in S^{\prime}$ cannot be contained in a single open half plane in $\mathbb{R}^{2}$. Thus, the conclusion of Lemma 4 is equivalent to there being $\hat{v}, \hat{w} \in S^{\prime}$ and constants $\lambda_{0} \geq 0, \lambda_{1}>0, \lambda_{2}>0$ such that $\lambda_{0} \nabla_{r} F(\hat{r})-\lambda_{1} \nabla_{r} G_{b}(\hat{r}, \hat{v})-\lambda_{2} \nabla_{r} G_{b}(\hat{r}, \hat{w})=0$. Therefore, we arrive at the following special case of a theorem of F. John:

Theorem 8 (F. John). Let $(0, b) \in E_{m}$ and let $\hat{r}=\hat{r}(b)$ be a solution of the extremal problem (**) above. Then, there exist points $\hat{v}, \hat{w} \in S_{b}$ and real numbers $\lambda_{0} \geq 0, \lambda_{1}>0, \lambda_{2}>0$ satisfying the following two conditions:

1. $G_{b}(\hat{r}, \hat{v})=0, G_{b}(\hat{r}, \hat{w})=0$.

2. The function $\Phi_{b}(r)=\lambda_{0} F(r)-\lambda_{1} G_{b}(r, \hat{v})-\lambda_{2} G_{b}(r, \hat{w})$ has a critical point at $\hat{r}$.

Now, we apply this theorem to the Thullen domain $E_{m}$ with $m>1$ at the reference point $(0, b)$ with $0<b<2^{-1 / 2 m}$. Recalling the preceding discussions on the (strong) square-concavity of the Kobayashi curves, we obtain the following key lemma, which is complementary to Lemma 3 :

Lemma 5. For every $b \in \mathbb{C}$ with $|b|<2^{-1 / 2 m}$, the unit sphere of the Wu metric in $T_{(0, b)} E_{m}$ does not meet the lower curve of the Kobayashi indicatrix. Moreover, the contact point between the $W u$ ellipse and the upper $K$-curve $C_{u p}$ in the first quadrant of $\mathbb{R}^{2}$ is unique and is not on the $v_{2}$-axis.

Proof. We use the square-concavity of the K-curves here. After the affine rescaling as in the paragraph preceding Lemma 3 followed by the change of variables to squares as before, the first claim reduces to proving the following simple statement: In the first quadrant of the xy-plane, consider the continuous function defined by

$$
g(x)= \begin{cases}g_{1}(x) & \text { if } 0<x<1-e_{1}, \\ -x+1 & \text { if } 1-e_{1}<x<1 \\ 0 & \text { otherwise, }\end{cases}
$$

where $e_{1}$ is a constant such that $0<e_{1}<1 / 2$. Assume also that $g$ is $C^{2}$ smooth and $g_{1}^{\prime \prime}(x)<0$ for all $0<x<1-e_{1}$. Then the smallest possible area of the triangle bounded by the axes and a straight line which stays above the graph of $y=g(x)$ is smaller than $1 / 2$.

In fact, a triangular area smaller than $1 / 2$ containing the area under the graph of $y=g(x)$ is easily attained by a straight line passing through the point $(1-g(1 / 2), g(1 / 2))$ with slope slightly larger than -1 , since $g(1 / 2)<1 / 2$. This shows that the minimum triangular area enclosed by a straight line segment together with the coordinate axes, which corresponds to the volume of the best fitting ellipsoid, is achieved by a straight line different from the one obtained by extending the straight line corresponding to the lower K-curve. We now use the fact that the whole curve corresponding to the Kobayashi indicatrix is $C^{1}$ smooth and squareconcave (Proposition 4) to conclude that the line segment minimizing the triangular area must in fact be part of the tangent line to the upper K-curve at a certain point $\hat{v}$. The strong square-concavity of the upper K-curve then implies that $\hat{v}$ is unique and does not lie on the $v_{2}$-axis. 
Now we are ready to prove the real analytic dependence of the Wu metric in the variable $b$ in the range $0<b<2^{-1 / 2 m}$.

First we consider the two conditions in the conclusion of Theorem 8 in our special setting. Let us denote by $\hat{r}=\hat{r}(b)$ the solution of extremal problem $(* *)$. Then, we set

$$
S_{b}=\left\{\left(v_{1}, v_{2}\right) \in \mathbb{R}^{2} \mid K(b, v)=1\right\} .
$$

Suppose that $\hat{v}=\left(\hat{v}_{1}, \hat{v}_{2}\right)$ and $\hat{w}=\left(\hat{w}_{1}, \hat{w}_{2}\right)$ are points satisfying the conditions in Theorem 8; then by Lemma 5 and the symmetry of $G_{b}(r, v)$ we have $\hat{v}_{1}^{2}=\hat{w}_{1}^{2}$ and $\hat{v}_{2}^{2}=\hat{w}_{2}^{2}$. By condition (2) of Theorem 8, this implies that

$$
\begin{aligned}
& \hat{\lambda} \hat{r}_{2}=\hat{v}_{1}^{2}, \\
& \hat{\lambda} \hat{r}_{1}=\hat{v}_{2}^{2},
\end{aligned}
$$

where $\hat{\lambda}>0$. (Although Theorem 8 only guarantees that $\hat{\lambda} \geq 0$, it is easy to see that $\hat{\lambda}$ cannot be zero by Lemma 5.) Also by Lemma 5 , we see that at the point $\hat{v}$ the Kobayashi indicatrix is real analytic and the tangent line of the upper K-curve coincides with the tangent line of the Wu ellipse. Thus, if we denote

$$
E(r, v)=r_{1} v_{1}^{2}+r_{2} v_{2}^{2}-1
$$

we have

$$
\begin{aligned}
E(\hat{r}, \hat{v}) & =0 \\
K(b, \hat{v}) & =1 \\
\hat{\mu} \frac{\partial K}{\partial v_{j}}(b, \hat{v}) & =\frac{\partial E}{\partial v_{j}}(\hat{r}, \hat{v}) \quad(j=1,2),
\end{aligned}
$$

where $\hat{\mu}>0$.

Note that (18), (19) and (21) imply

$$
\hat{r}_{1} \hat{v}_{1}^{2}=\hat{r}_{2} \hat{v}_{2}^{2}=\frac{1}{2}
$$

In turn, (23) is equivalent to the pair of equations

$$
\hat{\mu} \hat{v}_{j} K_{v_{j}}(b, \hat{v})=2 \hat{r}_{j} \hat{v}_{j}^{2}=1(j=1,2)
$$

where the variable subscript $v_{j}$ represents the partial derivative with respect to $v_{j}$. In summary, we now arrive at

$$
\left\{\begin{array}{l}
2 \hat{r}_{1} \hat{v}_{1}^{2}-1=0 \\
2 \hat{r}_{2} \hat{v}_{2}^{2}-1=0 \\
\hat{\mu} \hat{v}_{1} K_{v_{1}}(b, \hat{v})-1=0 \\
\hat{\mu} \hat{v}_{2} K_{v_{2}}(b, \hat{v})-1=0 \\
K(b, \hat{v})-1=0
\end{array}\right.
$$


Let $V=\left\{b \in \mathbb{R} \mid 0<b<2^{-1 / 2 m}\right\}$ and define the mapping $H: V \times(0, \infty)^{5} \rightarrow \mathbb{R}^{5}$ by

(24) $H\left(b, r_{1}, r_{2}, v_{1}, v_{2}, \mu\right)=\left(2 r_{1} v_{1}^{2}-1,2 r_{2} v_{2}^{2}-1, \mu v_{1} K_{v_{1}}-1, \mu v_{2} K_{v_{2}}-1, K-1\right)$.

Notice that $H$ is real analytic near the point $(b, \hat{r}, \hat{v}, \hat{\mu})$. Now, a direct computation shows that the Jacobian minor of $H$ with respect to the variables $r_{1}, r_{2}, v_{1}, v_{2}, \mu$ of the mapping is nonzero at $\left(\hat{r}_{1}, \hat{r}_{2}, \hat{v}_{1}, \hat{v}_{2}, \hat{\mu}\right)$ if and only if

$$
\operatorname{det}\left\{\left(\begin{array}{cc}
\hat{v}_{1}^{2} K_{v_{1} v_{1}} & \hat{v}_{1} \hat{v}_{2} K_{v_{1} v_{2}} \\
\hat{v}_{1} \hat{v}_{2} K_{v_{2} v_{1}} & \hat{v}_{2}^{2} K_{v_{2} v_{2}}
\end{array}\right)+\left(\begin{array}{cc}
\hat{v}_{1} K_{v_{1}} & 0 \\
0 & \hat{v}_{2} K_{v_{2}}
\end{array}\right)\right\} \neq 0
$$

at the point $(b, \hat{r}, \hat{v}, \hat{\mu}) \in V \times(0, \infty)^{5}$. Using the expression (3) in Section 3.1, we can conclude that the Kobayashi metric is in fact strongly convex and $K_{v_{j}}>0$ for $j=1,2$. This, together with the fact that $\hat{v}_{1}$ and $\hat{v}_{2}$ are non-zero (by Lemma $5)$, shows us that (25) indeed holds. Since $(* *)$ implies that $H(b, \hat{r}, \hat{v}, \hat{\mu})=0$, by the Implicit Function Theorem, locally there exists a uniquely determined triple of real analytic functions $\hat{r}(b), \hat{v}(b)$ and $\hat{\mu}(b)$ such that $H(b, \hat{r}(b), \hat{v}(b), \hat{\mu}(b))=0$. In particular, $\hat{r}=\hat{r}(b)$ is real analytic for $0<b<2^{-1 / 2 m}$. This proves the real analyticity of the Wu metric along the line $L=\left\{(0, b) \mid 0<b<2^{-1 / 2 m}\right\}$.

Now, recalling the definition of the open region $M^{-}$which admits a real analytic action of Aut $E_{m}$ whose orbits are transverse to the line $L$, and citing the invariance of the $\mathrm{Wu}$ metric under the action of automorphisms of $E_{m}$, we can immediately conclude that the $\mathrm{Wu}$ metric tensor is real analytic on $M^{-}$. This concludes the discussion on the smoothness of the Wu metric on $M^{-}$.

As mentioned in the beginning of the section, we now present an explicit description of the $\mathrm{Wu}$ metric tensor of $E_{m}$ at the points of $M^{-}$exploiting Lemma 5 in Section 3.4.2 together with Proposition 4 of Section 3.2.

Theorem 9. The Wu metric tensor of the Thullen domain $E_{m}$ for $m>1$ at the point $(0, b)$ with $0<b<2^{-1 / 2 m}$ is given by

$$
\begin{aligned}
& \frac{t^{2}}{2 t^{2 m} b^{2 m}\left((-m+1)+m t^{2}-t^{2 m} b^{2 m}\right)} d z^{1} \otimes \overline{d z^{1}} \\
& +\frac{m^{2} t^{2}}{2 b^{2}\left((-m+1)+m t^{2}-t^{2 m} b^{2 m}\right)^{2}} d z^{2} \otimes \overline{d z^{2}}
\end{aligned}
$$

where $t$ is the unique solution to the equation

$$
t^{2}+(m-2) t^{2 m} b^{2 m}-(m+1) t^{2 m+2} b^{2 m}+2 t^{4 m} b^{4 m}=0
$$

satisfying $1<t<1 / b$.

Proof. Fix a point $(0, b)$, with $0<b<2^{-1 / 2 m}$. By Lemma 5 , the ellipse in $\mathbb{R}^{2}$, which corresponds to the unit sphere of the $\mathrm{Wu}$ metric, is tangent to the upper $\mathrm{K}$-curve at exactly one point in the first quadrant of $\mathbb{R}^{2}$. Under the change of coordinates $\left(v_{1}, v_{2}\right) \mapsto\left(v_{1}^{2}, v_{2}^{2}\right)$ of $\mathbb{R}^{2}$, this ellipse becomes a straight line, which we call $\ell$ momentarily, as observed earlier. This line $\ell$ is tangential to the transformation of the upper K-curve whose parametric equation $(x(t), y(t))$ is given in (10) of Section 3.2 , and of course the line $\ell$ and the curve $(x(t), y(t))$ share no other intersection 
than the common tangent point, which we denote by $\left(x\left(t_{1}\right), y\left(t_{1}\right)\right)$. Then, the $v_{1^{-}}$ and $v_{2}$-intercepts of the line $\ell$ are

$$
\frac{-y\left(t_{1}\right) x^{\prime}\left(t_{1}\right)+y^{\prime}\left(t_{1}\right) x\left(t_{1}\right)}{y^{\prime}\left(t_{1}\right)}
$$

and

$$
\frac{y\left(t_{1}\right) x^{\prime}\left(t_{1}\right)-y^{\prime}\left(t_{1}\right) x\left(t_{1}\right)}{x^{\prime}\left(t_{1}\right)}
$$

respectively. Since the line $\ell$ together with the coordinate axes encloses the smallest triangular area in the first quadrant when compared to other tangent lines of $(x(t), y(t))$, by considering the derivative of the area functional we can conclude that $t=t_{1}$ satisfies the following equation:

$$
\left[y(t) x^{\prime}(t)-y^{\prime}(t) x(t)\right]\left[y(t) x^{\prime}(t)+y^{\prime}(t) x(t)\right]\left[x^{\prime}(t) y^{\prime \prime}(t)-x^{\prime \prime}(t) y^{\prime}(t)\right]=0 .
$$

In Section 3.2, we have shown that

$$
x^{\prime}(t)<0, \quad y^{\prime}(t)>0, \quad y(t) x^{\prime}(t)-y^{\prime}(t) x(t)<0,
$$

and

$$
x^{\prime}(t) y^{\prime \prime}(t)-x^{\prime \prime}(t) y^{\prime}(t)>0
$$

for all $1<t<1 / b$. Therefore, the condition for $t_{1}$ now becomes

$$
y\left(t_{1}\right) x^{\prime}\left(t_{1}\right)+y^{\prime}\left(t_{1}\right) x\left(t_{1}\right)=0,
$$

or equivalently, using the parameterization (10),

$$
t_{1}^{2}+(m-2) t_{1}^{2 m} b^{2 m}-(m+1) t_{1}^{2 m+2} b^{2 m}+2 t_{1}^{4 m} b^{4 m}=0 .
$$

The intersections of $L$ with the coordinate axes in the first quadrant can now be expressed in terms of $t_{1}$ and $b$, and the coefficients of the Wu metric tensor are then easily computed as asserted.

Again, we remark that the smoothness of the $\mathrm{Wu}$ metric tensor on $\mathrm{M}^{-}$can also be obtained by applying the Implicit Function Theorem to the conclusion of Theorem 9 above. Such a proof could be slightly simpler than using the functional $H$, but it is not helpful when it comes to the investigation of the smoothness of the Wu metric on the thin sets $M^{0}$ and $Z$.

3.4.3. Wu metric at $\left(p_{1}, p_{2}\right) \in Z$. First, notice that the K-curve in this case is solely given by a natural extension of the formula (3) in Section 3.1, since the lower K-curve represented by (2) shrinks to a single point. Furthermore, at $(0,0)$ the formula (3) yields that the set $k(v)=1$ is represented by the equation

$$
v_{1}^{2}+v_{2}^{2 m}=1
$$

in the first quadrant of $\mathbb{R}^{2}$. Notice that this curve is clearly square-concave, and is strictly square-concave away from the $v_{2}$-axis. Following $[6]$, one can deduce that the lower K-curve at $(0, b) \in E_{m}$ with $-2^{-1 / 2 m}<b<2^{-1 / 2 m}$ depends on the real 
variable $b$, is real analytic if $m>1$ is an integer, and $C^{[2 m]}$ smooth if $m>1$ is not an integer. Here, $[2 m]$ represents the largest integer which is not larger than $2 m$. Also, it is easy to verify that the contact point between the K-curve described by (29) and the ellipse representing the $\mathrm{Wu}$ metric sphere is unique and stays away from the $v_{2}$-axis. By the continuity of the $\mathrm{Wu}$ metric (Proposition 2 ), and the smoothness (at least $C^{2}$ ) of the upper K-curve there exists a constant $\epsilon>0$ such that the contact point between the $\mathrm{K}$-curves and the $\mathrm{Wu}$ ellipse is unique and is on the upper K-curve at which the upper K-curve is strictly square-concave at every reference point $(0, b)$ with $-\epsilon<b<\epsilon$.

Therefore, we may now apply the Implicit Function Theorem to the function $H$ defined in (24), to obtain

Proposition 6. The Wu metric tensor of $E_{m}$ with $m>1$ is real analytic if $m$ is an integer, and $C^{[2 m]-1}$ smooth otherwise, at every point $(z, 0) \in E_{m}$ with $|z|<1$.

Proof. The proof of appropriate smoothness of the $\mathrm{Wu}$ metric tensor on the line $\{(0, b) \mid-\epsilon<b<\epsilon\}$ is given above. Notice that the proof and the conclusion are the same at points on the disk $U=\{(0, b)|b \in \mathbb{C}| b \mid,<\epsilon\}$. Now the assertion of the proposition follows from the real analytic action by Aut $E_{m}$ across $U$ along $Z$, similarly to the preceding cases.

3.4.4. Wu metric at $\left(p_{1}, p_{2}\right) \in M^{0}$. The primary goal of this section is to prove that the Wu metric is $C^{1}$ smooth at the point $\left(0,2^{-1 / 2 m}\right)$, which together with the preceding three sections will finally conclude the proof of Theorem 2 .

We begin by showing that the Kobayashi indicatrix at every point is indeed $C^{2}$ smooth in each tangent plane. The $C^{1}$ smoothness is shown in [6], but no investigation on further differentiability was given, mainly due to the complexity of their computation. Here, we present a thorough conclusion.

Proposition 7. Let $m>1$ and let $(0, b) \in E_{m}$ be fixed, for some $b>0$. Then the Kobayashi indicatrix is globally $C^{2}$ smooth, but not $C^{3}$ smooth.

Proof. The proof follows from a simple implicit differentiation, using our parametric equation (10) introduced earlier. To avoid stating trivialities, we will simply sketch the proof here. First, write as $v_{2}=f_{\text {up }}\left(v_{1}\right)$ and $v_{2}=f_{\text {low }}\left(v_{1}\right)$ respectively the equations for the upper and lower $\mathrm{K}$-curves in $\mathbb{R}^{2}$ for the Kobayashi indicatrix in the tangent space $\mathbb{C}^{2}=T_{(0, b)} E_{m}$ as before. Again it is enough to check the smoothness of the whole K-curve (upper and lower curves combined) at the "joining point." (See the paragraph preceding Lemma 3 in Section 3.4.1 for this terminology.) Now consider the parametric equation (10) considered earlier in the context of the square-concavity of the Kobayashi curves. It is elementary to see that the following statement is valid:

When evaluated at the joining point of the upper and the lower K-curves, we have $d^{\ell} f_{\text {up }} / d v_{1}^{\ell}=d^{\ell} f_{\text {low }} / d v_{1}^{\ell}$ for $\ell=1, \ldots, p$ if and only if $\left(d / d x_{1}\right)^{\ell} x_{2}$ for the upper K-curve coincides with that for the lower K-curve for every $\ell=1, \ldots, p$.

Notice that the value of $d^{\ell} x_{2} / d x_{1}{ }^{\ell}$ for the lower K-curve is identically zero for every $\ell \geq 2$. But it is easy to check that for the upper K-curve, the value of $d^{\ell} x_{2} / d x_{1}^{\ell}$ is zero for $\ell=2$ while nonzero for $\ell=3$. Therefore, the Kobayashi indicatrix at the joining point between the upper and lower pieces is $C^{2}$ smooth but not $C^{3}$ smooth. 
Now we are ready to establish

Proposition 8. The Wu metric for the Thullen domain $E_{m}$ with $m>1$ is $C^{1}$ smooth at each point $z \in M^{0}=\left\{f\left(0,2^{-1 / 2 m}\right) \mid f \in\right.$ Aut $\left.E_{m}\right\}$.

Proof. This proposition follows again from the arguments above involving the Implicit Function Theorem on Fritz John's method with a few adjustments: First, the contact points between the K-curve and the best fitting ellipse may no longer be unique. But, the set of contact points is always connected due to the squareconcavity of the K-curve. By a careful application of the Intermediate Value Theorem on the gradients of the volume function and the constraints, one can choose a new contact point so that the same functional $H$ defined in (24) for the Implicit Function Theorem arguments can be used as before. Notice however that the functional involves the first derivative of the K-curve. Therefore, the Implicit Function Theorem yields the $C^{1}$ smoothness of the $\mathrm{Wu}$ metric at the points in $M^{0}$.

We remark that the choice of the special contact points in the proof can also be made to be a $C^{1}$ smooth function of the reference points, as a consequence of the Implicit Function Theorem. More importantly, we remark that in general $C^{2}$ smoothness of the $\mathrm{Wu}$ metric at points of $M^{0}$ cannot be obtained. As an application of our analysis of the holomorphic curvature of the Wu metric in the following section, we will explicitly show in Appendix A at the end of the paper that $C^{2}$ smoothness of the $\mathrm{Wu}$ metric is unattainable across $M^{0}$ in $E_{2}$.

\section{Holomorphic Curvature of the Wu metric}

4.1. Notation and generalities. Let $g$ be a $C^{2}$ Hermitian metric on a complex manifold $M$ with $g=\sum g_{i \bar{j}} d z^{i} d \bar{z}^{j}$ in local coordinates. Then the coefficients of the curvature tensor are given by :

$$
R_{i \bar{\jmath} k \bar{\ell}}=-\frac{\partial^{2} g_{i \bar{\jmath}}}{\partial z^{k} \partial \bar{z}^{\ell}}+\sum g^{\alpha \bar{\beta}} \frac{\partial g_{i \bar{\beta}}}{\partial z^{k}} \frac{\partial g_{\alpha \bar{\jmath}}}{\partial \bar{z}^{\ell}} .
$$

The holomorphic curvature at a point $p$ in the direction $\left(\zeta^{1}, \zeta^{2}, \ldots, \zeta^{n}\right)$ is

$$
\frac{\sum R_{i \bar{\jmath} k \bar{\ell}}(p) \zeta^{i} \bar{\zeta}^{j} \zeta^{k} \bar{\zeta}^{\ell}}{\sum g_{i \bar{\jmath}}(p) g_{k \bar{\ell}}(p) \zeta^{i} \bar{\zeta}^{j} \zeta^{k} \bar{\zeta}^{\ell}} .
$$

The holomorphic curvature is said to be bounded from above by a negative constant $c$, if

$$
\sum R_{i \bar{\jmath} k \bar{\ell}}(p) \zeta^{i} \bar{\zeta}^{j} \zeta^{k} \bar{\zeta}^{\ell}<c \sum g_{i \bar{\jmath}}(p) g_{k \bar{\ell}}(p) \zeta^{i} \bar{\zeta}^{j} \zeta^{k} \bar{\zeta}^{\ell}
$$

for every vector $\left(\zeta^{1}, \zeta^{2}, \ldots, \zeta^{n}\right)$. (See [15] for instance.) If $g$ is only continuous, the above definition can still have a well-defined meaning as explained in [19] and [20]: the holomorphic curvature of $g$ is bounded from above by a negative constant $c$ in the sense of current, if for every embedded Riemann surface $S \subset M$ the inequality $\partial \bar{\partial} \log G \geq-(c / 2) G d z \wedge d \bar{z}$ holds in the sense of current, where $\left.g\right|_{S}=G d z d \bar{z}$. If $g$ is $C^{2}$, the two notions of negativity introduced here obviously coincide.

It is not known in general what bounded domains admit an invariant Hermitian metric whose holomorphic curvature is negative everywhere. Partial answers are known for the Einstein-Kähler metric ([5]) and for the Bergman metric ([2]) of a 
Thullen domain $E_{m}$ with $m>1$. In this section, we shall prove Theorems 3 and 4, which show comprehensively the behavior of the holomorphic curvature of the Wu metric of the Thullen domains $E_{m}$ for all real numbers $m \geq 1 / 2$. Again, the case $m=1$ is excluded since the Thullen domain $E_{1}$ is the unit ball in $\mathbb{C}^{2}$ and the $\mathrm{Wu}$ metric then coincides with the normalized Poincaré-Bergman metric. Now we proceed with a detailed analysis of the holomorphic curvature of the Wu metric.

4.2. Holomorphic curvature on $E_{m}$ with $1 / 2<m<1$. Due to the isometric action by the automorphism group for the domain $E_{m}$ as pointed out in the preceding sections, it suffices to investigate the properties of the holomorphic curvature tensor at the point $(0, b)$, with $0<b<1$. Using the explicit formula of the $\mathrm{Wu}$ metric for $E_{m}, 1 / 2 \leq m<1$, given in Theorem 6 in Section 3.3, one can easily check that:

$$
\begin{aligned}
h_{1 \overline{1}}(0, b) & =\frac{1}{1-b^{2 m}}, \\
h_{1 \overline{2}}(0, b) & =h_{2 \overline{1}}(0, b)=0, \\
h_{2 \overline{2}}(0, b) & =\frac{1}{\left(1-b^{2}\right)^{2}},
\end{aligned}
$$

and

$$
\begin{aligned}
& R_{1 \overline{1} 1 \overline{1}}(0, b)=\frac{-1}{\left(1-b^{2 m}\right)^{2}}+\frac{-b^{2}}{\left(1-b^{2}\right)^{2} m^{2}}-\frac{1}{1-b^{2 m}} \\
& R_{1 \overline{1} 2 \overline{2}}(0, b)=\frac{-b^{2 m} m^{2}}{\left(1-b^{2 m}\right)^{3} b^{2}} \\
& R_{2 \overline{2} 1 \overline{1}}(0, b)=\frac{-1-b^{2}}{\left(1-b^{2}\right)^{3} m}+\frac{\left(1-b^{2 m}\right) b^{2}}{\left(1-b^{2}\right)^{4} m^{2}} \\
& R_{1 \overline{2} 2 \overline{1}}(0, b)=R_{2 \overline{1} 1 \overline{2}}(0, b)=\frac{-b^{2}-1}{\left(1-b^{2}\right)^{3} m}+\frac{b^{2 m}}{\left(1-b^{2 m}\right)\left(1-b^{2}\right)^{2}} \\
& R_{2 \overline{2} 2 \overline{2}}(0, b)=\frac{-2}{\left(1-b^{2}\right)^{4}}
\end{aligned}
$$

All the other terms vanish. So we have

$$
\begin{gathered}
\sum R_{i \bar{\jmath} k \bar{\ell}}(0, b) \zeta^{i} \bar{\zeta}^{j} \zeta^{k} \bar{\zeta}^{\ell}=\left(\frac{-1}{\left(1-b^{2 m}\right)^{2}}+\frac{-b^{2}}{\left(1-b^{2}\right)^{2} m^{2}}-\frac{1}{1-b^{2 m}}\right)\left|\zeta^{1}\right|^{4} \\
+\left(\frac{-b^{2 m} m^{2}}{\left(1-b^{2 m}\right)^{3} b^{2}}+\frac{-3 b^{2}-3}{\left(1-b^{2}\right)^{3} m}+\frac{2 b^{2 m}}{\left(1-b^{2 m}\right)\left(1-b^{2}\right)^{2}}+\frac{\left(1-b^{2 m}\right) b^{2}}{\left(1-b^{2}\right)^{4} m^{2}}\right)\left|\zeta^{1}\right|^{2}\left|\zeta^{2}\right|^{2} \\
+\frac{-2}{\left(1-b^{2}\right)^{4}}\left|\zeta^{2}\right|^{4}
\end{gathered}
$$

At this stage, we note that

$$
m b^{m}\left(1-b^{2}\right)<1-b^{2 m}<m\left(1-b^{2}\right) b^{m-1}
$$

whenever $0<b<1$ and $0<m<1$. This can be easily verified by checking the 
first and second derivatives in the variable $b$. Using these inequalities, we have

$$
\begin{aligned}
& \frac{-b^{2 m} m^{2}}{\left(1-b^{2 m}\right)^{3} b^{2}}+\frac{2 b^{2 m}}{\left(1-b^{2 m}\right)\left(1-b^{2}\right)^{2}}+\frac{-3 b^{2}-3}{\left(1-b^{2}\right)^{3} m}+\frac{\left(1-b^{2 m}\right) b^{2}}{\left(1-b^{2}\right)^{4} m^{2}} \\
& \quad<\frac{-b^{2 m} m^{2}}{\left(1-b^{2 m}\right)^{3} b^{2}}+\frac{2 b^{2 m}}{\left(1-b^{2 m}\right)\left(1-b^{2}\right)^{2}}+\frac{-3 b^{2}-3}{\left(1-b^{2}\right)^{3} m}+\frac{b^{m+1}}{\left(1-b^{2}\right)^{3} m} \\
& \quad<\frac{-m^{2} b^{2 m}}{\left(1-b^{2 m}\right)^{3} b^{2}}+\frac{2}{\left(1-b^{2 m}\right)\left(1-b^{2}\right)^{3} m}\left[-\left(1-b^{2 m}\right)+m b^{2 m}\left(1-b^{2}\right)\right]<0 .
\end{aligned}
$$

This means that

$$
\begin{aligned}
\sum R_{i \bar{\jmath} k \bar{\ell}}(0, b) \zeta^{i} \bar{\zeta}^{j} \zeta^{k} \bar{\zeta}^{\ell} & \\
& \leq\left(\frac{-1}{\left(1-b^{2 m}\right)^{2}}+\frac{-b^{2}}{\left(1-b^{2}\right)^{2} m^{2}}-\frac{1}{1-b^{2 m}}\right)\left|\zeta^{1}\right|^{4}+\frac{-2}{\left(1-b^{2}\right)^{4}}\left|\zeta^{2}\right|^{4} \\
& \leq-\frac{1}{2} \sum h_{i \bar{\jmath}}(0, b) h_{k \bar{\ell}}(0, b) \zeta^{i} \bar{\zeta}^{j} \zeta^{k} \bar{\zeta}^{\ell} .
\end{aligned}
$$

Therefore, the curvature property in the conclusion of Theorem 3 follows.

Since the $\mathrm{Wu}$ metric is at least in the Hölder class $C^{1,[2 m]-1}$ at all the points $(z, 0) \in E_{m}$ in the domain, together with the arguments of Appendix B, the result of Theorem 3 implies

Corollary 1. The holomorphic curvature of the Wu metric on a Thullen domain $E_{m}$ with $1 / 2 \leq m<1$ is bounded from above by $-1 / 2$ everywhere in the sense of currents.

Moreover, from the explicit computation of the components of the curvature tensor above, one immediately obtains

Corollary 2. The Wu metric on $E_{m}$ with $1 / 2 \leq m<1$ is not Kähler anywhere.

4.3. Holomorphic curvature on $E_{m}$ with $m>1$. Based upon the explicit description of the Wu metric of $E_{m}$ given in Section 3.4, we will present two separate sections of analysis of the holomorphic curvature tensor of the Wu metric in the following: first at points of $M^{+}$, and then at points in $M^{-}$. For the notation, see Section 1.2.

4.3.1. Holomorphic curvature at points of $M^{+}$. We only need to consider the points $(0, b)$ in $E_{m}$, for $m>1$ and $b>2^{-1 / 2 m}$. Since an explicit formula for the Wu metric is given in Theorem 7 , one can easily check that

$$
\begin{aligned}
& R_{1 \overline{1} 1 \overline{1}}(0, b)=-2 g_{1 \overline{1}}(0, b) g_{1 \overline{1}}(0, b), \\
& R_{2 \overline{2} 2 \overline{2}}(0, b)=-2 g_{2 \overline{2}}(0, b) g_{2 \overline{2}}(0, b), \\
& R_{1 \overline{2} 2 \overline{1}}(0, b)=R_{2 \overline{1} 1 \overline{2}}(0, b)=R_{1 \overline{1} 2 \overline{2}}(0, b)=R_{2 \overline{2} 1 \overline{1}}(0, b)=-g_{1 \overline{1}}(0, b) g_{2 \overline{2}}(0, b), \\
& \text { All the remaining terms } R_{i \bar{\jmath} k \bar{\ell}}(0, b)=-g_{i \bar{\jmath}}(0, b) g_{k \bar{\ell}}(0, b)=0 .
\end{aligned}
$$

It follows immediately that the $\mathrm{Wu}$ metric has constant holomorphic curvature -2 along every holomorphic direction at $(0, b)$, whenever $b>2^{-1 / 2 m}$. Due to its invariance under the action of the automorphisms, the holomorphic curvature is identically equal to the constant -2 at every point in $G_{m}(b)$. This completes the proof of Theorem 4. 
4.3.2. Holomorphic curvature at points of $M^{-}$. The arguments of this section and the preceding section will complete the proof of Theorem 4 . We will show the negativity of the holomorphic curvature at every point of $M^{-}$by a direct computation. Since the computation is very tedious, we will only outline the main steps here. Again, notice that it is enough to show the negativity of the holomorphic curvature at a typical point $(0, b)$ with $0<b<2^{-1 / 2 m}$.

Recall that the Wu metric tensor $h_{i \bar{\jmath}}$ at $(0, b)$ is a diagonal matrix with respect to the Euclidean coordinates as explicitly computed in Theorem 9. Thus we write the $\mathrm{Wu}$ metric tensor at $(0, b)$ as

$$
h=h_{1}(0, b) d z_{1} \otimes \overline{d z_{1}}+h_{2}(0, b) d z_{2} \otimes \overline{d z_{2}} .
$$

By Theorem 9,

$$
h_{1}=\frac{1}{2} t^{2-2 m} b^{-2 m} B^{-1}, \quad h_{2}=\frac{1}{2} m^{2} t^{2} b^{-2} B^{-2},
$$

where

$$
B=1-m+m t^{2}-t^{2 m} b^{2 m}
$$

and where $t$ is the unique solution in the open interval $(1,1 / b)$ of the algebraic equation (equation (27) in Theorem 9)

$$
t^{2}+(m-2) t^{2 m} b^{2 m}-(m+1) t^{2} t^{2 m} b^{2 m}+2 t^{4 m} b^{4 m}=0
$$

Notice that

$$
B=\left(1-(t b)^{2 m}\right)+m\left(t^{2}-1\right)>0
$$

for any $t$ and $b$ with $1<t<1 / b$. Before we carry out any further detailed estimates, we first point out that throughout this section we have

$$
0<b<2^{-1 / 2 m}
$$

A direct computation yields, at the point $(0, b)$,

$$
\begin{aligned}
& R_{1 \overline{1} 1 \overline{1}}=-\frac{b h_{1}^{\prime}}{2 m}-2 h_{1}-\frac{b^{2} h_{2}}{m^{2}}, \\
& R_{1 \overline{1} 2 \overline{2}}=-\frac{h_{1}^{\prime \prime}}{4}-\frac{h_{1}^{\prime}}{4 b}+\frac{\left(h_{1}^{\prime}\right)^{2}}{4 h_{1}}=-\frac{h_{1}}{4}\left(\log h_{1}\right)^{\prime \prime}-\frac{h_{1}^{\prime}}{4 b} \\
& R_{1 \overline{2} 2 \overline{1}}=R_{2 \overline{1} 1 \overline{2}}=-\frac{b h_{2}^{\prime}}{2 m}-\frac{h_{2}}{m}+\frac{b h_{1}^{\prime} h_{2}}{2 m h_{1}}=-\frac{h_{1}}{2 m b}\left(\frac{b^{2} h_{2}}{h_{1}}\right)^{\prime}, \\
& R_{2 \overline{2} 1 \overline{1}}=-\frac{b h_{2}^{\prime}}{2 m}-\frac{h_{2}}{m}+\frac{b^{2} h_{2}^{2}}{m^{2} h_{1}}, \\
& R_{2 \overline{2} 2 \overline{2}}=-\frac{h_{2}^{\prime \prime}}{4}+\frac{\left(h_{2}^{\prime}\right)^{2}}{4 h_{2}}-\frac{h_{2}^{\prime}}{4 b}=\frac{-h_{2}\left(\log h_{2}\right)^{\prime \prime}}{4}-\frac{h_{2}^{\prime}}{4 b}
\end{aligned}
$$

and all the other components vanish, where the "primes" represent the derivative in the real variable $b$. To show that each component term listed above is real and negative, we need the following lemma. 
Lemma 6. With the notation as above, we have:

(i) $h_{1}^{\prime}>0$,

(ii) $\left(\log h_{1}\right)^{\prime \prime}>0$,

(iii) $\left(\left(b^{2} h_{2}\right) / h_{1}\right)^{\prime}>0$,

(iv) $R_{2 \overline{2} 1 \overline{1}}<R_{1 \overline{2} 2 \overline{1}}$,

(v) $h_{2}^{\prime}>0$

(vi) $\left(\log h_{2}\right)^{\prime \prime} \geq 2\left(\log h_{1}\right)^{\prime \prime}$,

Lemma 6 will immediately imply that the holomorphic curvature is negative. Note that part (iv) of Lemma 6 also implies the following:

Corollary 3. The Wu metric of $E_{m}$ for $m>1$ is non-Kähler at every point of $M^{-}$.

To prove Lemma 6 , we need the following two lemmas.

Lemma 7. For $(0, b) \in E_{m}$ with $0<b<2^{-1 / 2 m}$ and for $t$ in Theorem 9, we have

$$
\frac{1}{m+1}<t^{2 m} b^{2 m}<1 / 2
$$

Proof. Since $1<t<1 / b$, we have $(t b)^{2 m}<1$ obviously. Regarding the algebraic equation (27) as a quadratic equation in $(t b)^{2 m}$ with coefficients involving $t$ and $m$ and using the properties of convex parabola, we obtain the inequalities

$$
0<(t b)^{2 m}<1 / 2
$$

Now, the equation (27) can be rewritten as

$$
\left(2 t^{2 m} b^{2 m}-1\right)\left(t^{2 m} b^{2 m}-1\right)=\left(t^{2}-1\right)\left((m+1) t^{2 m} b^{2 m}-1\right) .
$$

Since the left hand side is positive, so is the right hand side. Since $t>1$, we now get

$$
t^{2 m} b^{2 m}>\frac{1}{m+1}
$$

which completes the proof.

We also have

Lemma 8. Consider $t$ as the function of $b$ defined by the algebraic equation (27), for $0<b<2^{-1 / 2 m}$. Then,

$$
\frac{d t}{d b}<0
$$

Proof. Dividing both sides of the equation (27) by $t^{2}$, and differentiating in the variable $b$, we obtain

$$
\frac{d t}{d b}=\frac{M_{1}}{M_{2}}
$$

where

$$
\begin{aligned}
& M_{1}=m t\left(-m+2+(m+1) t^{2}-4 t^{2 m} b^{2 m}\right), \\
& M_{2}=-b\left(-m^{2}+3 m-2+m(m+1) t^{2}-2(2 m-1) t^{2 m} b^{2 m}\right) .
\end{aligned}
$$


By Lemma 7, one obtains

$$
\begin{aligned}
& M_{1} \geq m t\left(m\left(t^{2}-1\right)+t^{2}\right) \geq m t^{3}>1, \\
& M_{2} \leq-b\left(m^{2}\left(t^{2}-1\right)+m t^{2}+(m-1)\right) \leq-b m t^{2}<0 .
\end{aligned}
$$

This proves the lemma.

Proof of Lemma 6 . The inequalities in (i), (ii), (iii) and (v) can be obtained by direct computation using Lemma 7 and Lemma 8. From the expression of $R_{a \bar{b} c} \bar{d}$ given at the beginning of Section 4.3.2, we have ,

$$
\begin{aligned}
R_{1 \overline{2} 2 \overline{1}}-R_{2 \overline{2} 1 \overline{1}} & =\frac{b h_{2}}{m h_{1}}\left(\frac{h_{1}^{\prime}}{2}-\frac{b h_{2}}{m}\right) \\
& =\frac{m^{3} t^{2 m} b^{2 m}(m-1)\left(t^{2}-1\right)}{2 b B^{3}\left|M_{2}\right|}>0
\end{aligned}
$$

where the last equality is obtained from a direct computation together with exploiting (27) in the statement of Theorem 8. This proves part (iv).

To prove part (vi), we need to regroup terms appropriately. From the computation of $h_{1}$, we obtain

$$
\left(\log h_{1}\right)^{\prime \prime}=\frac{2 m}{B^{2} M_{2}^{2}} \cdot\left(Y_{1}+Y_{2}+Y_{3}\right)
$$

where

$$
\begin{aligned}
Y_{1} & =Q B \frac{\partial M_{2}}{\partial b}+Q B \frac{\partial M_{2}}{\partial t} \cdot \frac{M_{1}}{M_{2}}, \\
Y_{2} & =-B M_{1} \frac{\partial Q}{\partial t}+Q M_{1} \frac{\partial B}{\partial t} \\
Y_{3} & =-B M_{2} \frac{\partial Q}{\partial b}+Q M_{2} \frac{\partial B}{\partial b}, \\
Q & =(m-1) t^{2}\left(1-(t b)^{2 m}\right)+m\left(t^{2}-(t b)^{2 m}\right) .
\end{aligned}
$$

Lemmas 7 and 8 can again be used to prove that $Y_{1}>0$ and $Y_{2}>0$. Actually, we have

$$
Y_{2}>\frac{2(m-1) M_{1}(t b)^{2 m}}{t}\left\{m^{2}\left(t^{4}-1\right)+(2 m-1) t^{2}+t^{2}\left(1-(t b)^{2 m}\right)\right\}>0 .
$$

The term $Y_{3}$ is unfortunately negative. A calculation yields

$$
Y_{3}=\frac{2 m^{2} M_{2}}{b}\left(t^{4}-1\right)(m-1) t^{2 m} b^{2 m} .
$$

To prove that $Y_{2}+Y_{3}>0$ we will extract two positive terms from the expression (36) for $Y_{2}$, and add to $Y_{3}$. Namely we consider

$$
Y_{4}=\frac{2(m-1) M_{1}(t b)^{2 m}}{t}\left(m^{2}\left(t^{4}-1\right)+(2 m-1) t^{2}\right)+Y_{3} .
$$


Using inequality $(35)$ and $(t b)^{2 m}<1 / 2$, after a direct computation, we have

$$
Y_{4}>2(m-1)(t b)^{2 m}(2 m-1) m t^{4}>0
$$

This proves the lemma.

Remarks on strict negativity of holomorphic curvature. It turns out that the holomorphic curvature of the Wu metric at points of $M^{-}$is bounded from above by a negative constant. This can be obtained by showing that the limits of the holomorphic curvature as $b \rightarrow 0$ and $b \rightarrow 2^{-1 / 2 m}$, respectively, are bounded from above by a negative constant. Then of course by continuity and negativity of the holomorphic curvature mentioned above, one obtains the strict negativity of the holomorphic curvature of the $\mathrm{Wu}$ metric at points of $M^{-}$, thus proving the final assertion of Theorem 4. We choose not to include any further computation involving this to avoid repeating ourselves with tedious estimates.

\section{Appendix A: The Wu metric is $C^{1}$ But not $C^{2}$ ACross $M^{0}$}

Earlier, we have shown that the Wu metric of $E_{m}$ for $m>1$ is $C^{1}$ smooth at points of $M^{0}$. Here, we demonstrate that the Wu metric for $E_{2}$ is not $C^{2}$ smooth through a numerical calculation.

To see this, we first solve for $t$ in terms of $b$ explicitly in the equation in Theorem 9 :

$$
t=\frac{1}{b \sqrt{2 \cos \left(\left(\cos ^{-1}\left(-b^{2}\right)\right) / 3\right)}} .
$$

Using this in the expression of $R_{1 \overline{1} 2 \overline{2}}$ at $(0, b) \in M^{-}$and $(0, b) \in M^{+}$, and taking limits as $b / 2^{-1 / 4}$ and $b \searrow 2^{-1 / 4}$ respectively, one sees that the $\mathrm{Wu}$ metric is of class $C^{1}$ but not $C^{2}$ at the point $\left(0,2^{-1 / 4}\right)$, which belongs to $M^{0}$. While we choose not to include any further detailed computations here, we simply mention that a numerical computation on the limit gives the following:

$$
\lim _{b \nearrow\left(2^{-1 / 4}\right)} R_{1 \overline{1} 2 \overline{2}}(0, b)=-20.113 \cdots
$$

and

$$
\lim _{b \backslash\left(2^{-1 / 4}\right)} R_{1 \overline{1} 2 \overline{2}}(0, b)=-16 \sqrt{2} .
$$

This discrepancy implies that the Wu metric at points of $M^{0}$ is not $C^{2}$ smooth.

\section{Appendix B: Proof of Theorem 5}

We will begin with the following general situation with a function of two real variables.

Let $u(x, y)$ be a real-valued $C^{1}$ smooth function on a neighborhood of the closure of the disk $D=\left\{(x, y) \in R^{2} \mid x^{2}+y^{2}<1\right\}$, and let $\gamma$ be a piecewise $C^{1}$ smooth curve without self-intersection passing through the origin such that $D \backslash \gamma$ has exactly two connected components, say $D_{1}$ and $D_{2}$. Also assume that $\gamma$ meets $\partial D$ transversally. Then we have 
Lemma. If $u(x, y)$ is $C^{1}$ smooth on $D, C^{2}$ smooth on $D_{1} \cup D_{2}$, and satisfies

$$
\Delta u(x, y) \geq \operatorname{ch}(x, y) \quad \text { for all }(x, y) \in D_{1} \cup D_{2}
$$

for some constant $c>0$ and a $C^{1}$ function $h(x, y)$ defined on $D$, then for any smooth nonnegative real valued function $\varphi$ on $D$ with compact support we have

$$
\int_{D} u \Delta \varphi d A \geq c \int_{D} h \varphi d A
$$

In particular, we have

$$
\partial \bar{\partial} u \geq c \cdot h d z \wedge d \bar{z}
$$

on $D$, in the sense of current.

Proof. First set up the following notation for a $C^{1}$ smooth function $f(x, y)$ :

$$
\delta f=f_{x} d y-f_{y} d x .
$$

Notice that, in case $f$ is $C^{2}$ smooth,

$$
d \delta f=(\Delta f) d x \wedge d y .
$$

Therefore, for $u$ on $D_{j}(j=1,2)$, we now have

$$
\begin{aligned}
& d(u \delta \varphi)=\left(u_{x} \varphi_{y}+u_{y} \varphi_{y}\right) d x \wedge d y+u \Delta \varphi d x \wedge d y \\
& d(\varphi \delta u)=\left(u_{x} \varphi_{y}+u_{y} \varphi_{y}\right) d x \wedge d y+\varphi \Delta u d x \wedge d y
\end{aligned}
$$

Thus,

$$
\varphi \Delta u d x \wedge d y-u \Delta \varphi d x \wedge d y=d(\varphi \delta u-u \delta \varphi)
$$

on $D_{1}$ and also on $D_{2}$.

Now, apply Stokes' Theorem to this. Let $\varphi(x, y)$ be a $C^{\infty}$ smooth function with compact support in $D$. Then we have

$$
\begin{aligned}
\int_{D} u \Delta \varphi d x \wedge d y= & \int_{D_{1}} u \Delta \varphi d x \wedge d y+\int_{D_{2}} u \Delta \varphi d x \wedge d y \\
= & \int_{D_{1}} \varphi \Delta u d x \wedge d y+\int_{D_{2}} \varphi \Delta u d x \wedge d y \\
& +\int_{D_{1}} d(\varphi \delta u-u \delta \varphi)+\int_{D_{2}} d(\varphi \delta u-u \delta \varphi) .
\end{aligned}
$$

Since $\varphi$ does not vanish at every boundary point of $D_{1}$ or $D_{2}$, we need to handle the final two integrals with care.

By Stokes' Theorem, we have

$$
\begin{aligned}
\int_{D_{1}} d & (\varphi \delta u-u \delta \varphi)+\int_{D_{2}} d(\varphi \delta u-u \delta \varphi) \\
& =\int_{\partial D_{1}} \varphi \delta u-u \delta \varphi+\int_{\partial D_{2}} \varphi \delta u-u \delta \varphi \\
& =\int_{\partial D} \varphi \delta u-u \delta \varphi+\int_{\gamma} \varphi \delta u-u \delta \varphi+\int_{-\gamma} \varphi \delta u-u \delta \varphi \\
& =0
\end{aligned}
$$


Notice that an approximation of $\gamma$ followed by an application of a convergence theorem for line integrals is required to make the above arguments valid. But this is routine, as the integrand is continuous everywhere on the closure of $D$. Now,

$$
\begin{aligned}
\int_{D} u \Delta \varphi d x \wedge d y & =\int_{D_{1}} \varphi \Delta u d x \wedge d y+\int_{D_{2}} \varphi \Delta u d x \wedge d y \\
& \geq c \int_{D_{1}} \varphi h d x \wedge d y+\int_{D_{2}} \varphi h d x \wedge d y \\
& =c \int_{D} \varphi h d x \wedge d y,
\end{aligned}
$$

as desired.

To establish the proof of negativity of the holomorphic curvature current, first observe that the set $M^{0}$ at which the Wu metric is only $C^{1}$ smooth is explicitly described by the equation

$$
|z|^{2}+b^{-2 m}|w|^{2 m}=1
$$

which is strongly pseudoconvex at $(0, b)$, where $b=2^{-1 / 2 m}$. Thus any embedded analytic set through $(0, b)$ is either transversal to $M^{0}$ or tangential to $M^{0}$ with order of contact 2 , when restricted to a smaller variety through $(0, b)$ if necessary. One concludes therefore that the intersection between the analytic set and $M^{0}$ is either a single point or a piecewise smooth curve. Now, the lemma above together with Theorems 1 through 4 implies the negativity of the holomorphic curvature current at points on $M^{0}$. To complete the proof, we finally consider the points on the set $Z=\left\{(z, 0) \in \mathbb{C}^{2}|| z \mid<1\right\}$. It is obvious that any embedded closed analytic disk (which admits a holomorphic extension across the boundary) passing through $(0,0)$ can meet the set $Z$ along a discrete set of points only, unless it coincides with a subset of $Z$. When the embedded disk is transversal to $Z$, the problem reduces to the previous arguments using the lemma above. When the embedded disk is in fact coincides with an open subset of $Z$ containing the origin, the holomorphic curvature of the $\mathrm{Wu}$ metric in the direction of $Z$ is realized by the Gauss curvature of the $\mathrm{Wu}$ metric restricted to $Z$, since $Z$ is clearly a totally geodesic complex submanifold with respect to the $\mathrm{Wu}$ metric. Then the negativity of the holomorphic curvature in the direction of $Z$ follows immediately. This concludes the proof.

\section{REFERENCES}

1. L. Ahlfors, An extension of Schwarz's lemma, Trans. Amer. Math. Soc. 43 (1938), 359-364.

2. K. Azukawa and M. Suzuki, The Bergman metric on a Thullen domain, Nagoya Math. J. 89 (1983), 1-11. MR 84m:32030

3. E. Bedford and S. Pinchuk, Domains in $\mathbb{C}^{2}$ with noncompact holomorphic automorphism groups, Math. USSR Sbornik 63 (1989), 141-151. MR 89d:32054

4. S. Bergman, The kernel function and conformal mapping, (2nd ed.), Mathematical Surveys, No. 5, Amer. Math. Soc., Providence, R.I., 1970. MR 58:22502

5. J. Bland, The Einstein-Kähler metric on $\left\{|z|^{2}+|w|^{2 p}<1\right\}$, Michigan Math. J. 33 (1986), 209-220. MR 87i:32036

6. B. Blank, D. Fan, D. Klein, S. Krantz, D. Ma, and M. Pang, The Kobayashi metric of a complex ellipsoid in $\mathbb{C}^{2}$, Experimental Mathematics 1 (1992)), 47-55. MR 93h:32032

7. D. Burns, S. Shnider, R. Wells, On deformations of strictly pseudoconvex domains, Invent. Math. 46 (1978), 237-253. MR 58:1265 
8. S.Y. Cheng and S.T. Yau, On the existence of a complete Kähler metric on non-compact complex manifolds and the regularity of Fefferman's equation, Comm. Pure Appl. Math. 33 (1980), 507-544. MR 82f:53074

9. M. Jarnicki and P. Pflug, Invariant distance and metrics in complex analysis, Walter de Gruyter, Berlin, 1993. MR 94k:32039

10. F. John, Extremum problems with inequalities as subsidiary conditions, Studies and Essays Presented to Richard Courant, Interscience, New York (1948), 187-204. MR 10:719b

11. K. Kim, Domains in $\mathbb{C}^{n}$ with a piecewise Levi flat boundary which possess a noncompact automorphism group, Math. Ann. 292 (1992), 575-586. MR 93h:32024

12. K. Kim and J. Yu, Boundary behavior of the Bergman curvature in the strictly pseudoconvex polyhedral domains, Pacific J. Math., (to appear).

13. P. Klembeck, Kähler metrics of negative curvature, the Bergman metric near the boundary and the Kobayashi metric on smooth bounded strictly pseudoconvex sets, Indiana Univ. Math. J. (1978), 275-282. MR 57:3455

14. S. Kobayashi, Hyperbolic manifolds and holomorphic mappings, Marcel Dekker, New York, 1970. MR 43:3503

15. S. Kobayashi and K. Nomizu, Foundations of Differential Geometry, Volume II, Interscience Publishers, New York, 1969. MR 38:6501

16. L. Lempert, La métrique de Kobayashi et la representation des domains sur la boule, Bull. Soc. Math. France 109 (1981), 427-474. MR 84d:32036

17. Y.C. Lu, Holomorphic mappings of complex manifolds, J. Diff. Geom. 3 (1968), 292-313. MR 40:3482

18. B. Wong, Characterization of the unit ball in $\mathbb{C}^{n}$ by its automorphism group, Invent. Math 41 (1977), 253-257. MR 58:11521

19. H. Wu, A remark on holomorphic sectional curvature, Indiana Univ. Math. J. 22 (1972-1973), 1103-1108. MR 47:4191

20. Inst. 1987-88 (J.E. Fornaess ed.) Math. Notes, Princeton Univ. Press 38 (1993), 640-682. MR 94a:32038

21. Unpublished Notes.

22. S. T. Yau, A general Schwarz lemma for Kähler manifolds, American J. Math. 100 (1978), 197-203. MR 58:6370

Department of Mathematics, Boston College, Chestnut Hill, Massachusetts 02167

E-mail address: cheung/mt@hermes.bc.edu

Department of Mathematics, Pohang University of Science and Technology, Pohang, 790-784 South KoreA

E-mail address: kimkt@posmath.postech.ac.kr 